\title{
Stimulating TRPM7 Suppresses Cancer Cell Proliferation and Metastasis by Inhibiting Autophagy
}

Yanhong Xing

Xuzhou Medical University

Xiangqing Wei

Xuzhou Medical University

Yucheng Liu

Xuzhou Medical University

Meng-meng Wang

China Medical University

Yuan-hui Fei

Xuzhou Medical University

Zhongheng Sui

Xuzhou Medical University

Yang Zhang

Xuzhou Medical University

Yi Jiang

Xuzhou Medical University

Chen Lu

Xuzhou Medical University

Peng Zhang

Xuzhou Medical University

Rong Chen

Xuzhou Medical University

Nan Liu

Xuzhou Medical University

Mengmei Wu

Xuzhou Medical University

Lin Ding

Xuzhou Medical University

Yuqing Wang

Kyushu University: Kyushu Daigaku

Feng Guo 
China Medical University

Jiansong Qi

Dalhousie University

Wuyang Wang ( $\nabla$ wuyangwang80@gmail.com )

Xuzhou Medical University https://orcid.org/0000-0003-1875-5463

\section{Research}

Keywords: ion channels, TRPM7, autophagic arrest, zinc homeostasis, tumorigenesis, apoptosis, cell cycle, metastasis

Posted Date: July 6th, 2021

DOI: https://doi.org/10.21203/rs.3.rs-644549/v1

License: (a) This work is licensed under a Creative Commons Attribution 4.0 International License. Read Full License

Version of Record: A version of this preprint was published at Cancer Letters on November 1st, 2021. See the published version at https://doi.org/10.1016/j.canlet.2021.10.043. 


\section{Abstract}

Background-The transient receptor potential melastatin-subfamily member 7 (TRPM7) is a ubiquitous cation channel possessing kinase activity. TRPM7 mediates a variety of physiological responses by conducting flow of cations such as $\mathrm{Ca}^{2+}, \mathrm{Mg}^{2+}$, and $\mathrm{Zn}^{2+}$. TRPM7 has been reported to contribute to basal autophagy and tumorigenesis, however, its precise role in autophagy and functional effects on cancer still need to be elucidated.

Methods-We have utilized various TRPM7 modulators as well as the overexpression or knockdown of the TRPM7 channel to modulate TRPM7 activity in order to systematically define the role of TRPM7 in autophagy.

Results-Here, we show that the activation of TRPM7 channel stimulated by chemical agonists of TRPM7, Clozapine or Naltriben, inhibited autophagy via mediating $\mathrm{Zn}^{2+}$ release to the cytosol, presumably from the intracellular $\mathrm{Zn}^{2+}$-accumulating vesicles where TRPM7 localizes. $\mathrm{Zn}^{2+}$ release following the activation of TRPM7 disrupted the fusion between autophagosomes and lysosomes by disturbing the interaction between Sxt17 and VAMP8 which determines fusion status of autophagosomes and lysosomes. Ultimately, the disrupted fusion resulting from stimulation of TRPM7 channels arrested autophagy. Functionally, we demonstrate that the autophagy inhibition mediated by TRPM7 triggered cell death and suppressed metastasis of cancer cells in vitro, more importantly, restricted tumor growth and metastasis in vivo, by evoking apoptosis, cell cycle arrest, and reactive oxygen species (ROS) elevation.

Conclusion-We demonstrate that stimulating TRPM7 channels inhibits autophagy by disrupting the fusion between autophagosomes and lysosomes. Moreover, the TRPM7-induced autophagy inhibition suppresses cancer cell proliferation and metastasis by inducing apoptosis and cell cycle arrest. Our findings represent TRPM7 channels a great potential for cancer treatment.

\section{Background}

TRPM7 is a ubiquitously expressed cationic channel located on the plasma membrane that possesses serine/threonine kinase activity ${ }^{[1]}$. It is involved in a series of fundamental cellular processes and physiological responses and plays a role in embryonic development by conducting cationic flow including divalent $\mathrm{Ca}^{2+}, \mathrm{Mg}^{2+}$ and $\mathrm{Zn}^{2+}$ (zinc) as well as the monovalent ions $\mathrm{K}^{+}$and $\mathrm{Na}^{+[2,3]}$. Studies have shown that phosphatidylinositol 4,5-bisphosphate $\left(\mathrm{PI}(4,5) \mathrm{P}_{2}\right)$, intracellular $\mathrm{Mg}^{2+}$, and intracellular $\mathrm{pH}$ are endogenous modulators of TRPM7 channel activity ${ }^{[4-6]}$. Interestingly, emerging studies suggest that a vast majority of native TRPM7 is localized in the membrane of unknown intracellular vesicles where large quantities of zinc are stored ${ }^{[7]}$. This intracellular pool of TRPM7 senses oxidative stress and functions to buffer cytosolic zinc that in turn has catalytic, structural, and regulatory functions for proteins $^{[8]}$. 
Autophagy is a highly conserved catabolic process that controls quality of the cytoplasm by removing unwanted proteins and damaged organelles ${ }^{[9]}$. Autophagy is initiated when double membraned autophagosomes engulf cytosolic cargo destined for degradation, which is followed by the fusion of these vesicles with lysosomes and ultimate degradation of autophagic cargo by lysosomal hydrolases ${ }^{[10]}$. There is an increasing body of evidence indicating that autophagy plays a critical role in cascades involved in tumorigenesis ${ }^{[11]}$. Specifically, following tumor formation, malignant cells actively undergo autophagy to survive from limited nutrient supplies. In addition to this, studies have shown that inhibition of autophagy has been proposed as a strategy to combat cancer ${ }^{[12-14]}$. However, it remains debated whether autophagy is beneficial or detrimental to tumorigenesis.

TRPM7 has been reported to contribute to basal autophagy that is attributed to the regulation of $\mathrm{Ca}^{2+}$ entry ${ }^{[15]}$. In addition to this, TRPM7 is involved in regulating cell proliferation, cell cycle, and cell migration, likely owing to its kinase or channel activity ${ }^{[2,5,16-18]}$. A close interplay exists between the TRPM7 channel and cancer despite discrepancies among the published reports on TRPM7. Nevertheless, the role of TRPM7 in autophagy and the functional effect on cancer still needs to be elucidated.

Therefore, the objective of this study is to investigate the role of TRPM7 in autophagy and tumorigenesis.

Recently, several pharmacological compounds, including Clozapine, Naltriben, Proadifan, Mibefradil, FTY720, and NS8593, have been identified that either activate or inhibit TRPM7 activity ${ }^{[19,20]}$. These modulators are all drug-like molecules and are permeable across the cellular menbrane, which is supported by the properties that they modulate TRPM7 activity by affecting the gating of the channel[2022]. Experimental use of these compounds will allow us to explore the functional role of TRPM7.

To systematically explore the role of TRPM7 in autophagy and tumorigenesis and the underlying mechanisms responsible for these processes, we have utilized various TRPM7 modulators as well as the overexpression or knockdown of the TRPM7 channel to modulate TRPM7 activity in order to characterize the effects on autophagy. First, we demonstrate that activation of TRPM7 inhibits autophagy by disrupting the fusion between autophagosomes and lysosomes. Mechanistically, the activation of TRPM7 mediates intracellular zinc release to the cytosol, which in turn eliminates the interaction between two Soluble NSF Attachment Protein Receptor (SNARE) proteins, syntaxin 17 (Stx17) in the autophagosome and Vesicle-associated membrane protein 8 (VAMP8) in the lysosome ${ }^{[23]}$. This results in the disrupted fusion between autophagosomes and lysosomes that in turn arrests autophagic flux. Second, to further evaluate the antineoplastic effects of TRPM7, we used several cancer cell lines and in vivo xenograft and metastasis mouse models to investigate the effects of TRPM7-mediated autophagy inhibition on cancer cell proliferation and metastasis. We demonstrate that the autophagy inhibition mediated by TRPM7 suppresses tumor growth and metastasis both in vitro and in vivo by evoking apoptosis, cell cycle arrest, and ROS elevation. Collectively, these results indicate that TRPM7 controls oncogenic autophagy in cancer by mediating zinc release into the cytosol from intracellular stores.

\section{Materials And Methods}


Mammalian cell culture and transfection. Hela cells, HEK 293T cells, PaTu 8988t cells, and HPDE6c7 cells were purchased from Shanghai Gefan Biotechnology. Co., Ltd. MCF-7, MCF-10A, A-375, U-87 MG, SGC7901, and GES-1 were obtained from KeyGEN BioTECH. GFP-LC3 stably expressed HEK-293T cell was a gift from Dr. Ling Chen at Guangzhou Institutes of Biomedicine and Health, Chinese Academy Sciences. All cells were grown at $37^{\circ} \mathrm{C}$ in a $1: 1$ mixture of Dulbecco's modified Eagle's medium (DMEM) and F12 supplemented with $10 \%$ fetal bovine serum (FBS, Gibco) in a humidified 5\% CO2 incubator. Unless otherwise stated, experiments of cell viability measurement were performed with the following timeline: cells $(\sim 20 \times 104 / \mathrm{ml})$ were plated in medium with $10 \%$ FBS. After 24 hours, cells were changed to $2 \%$ FBS with or without drugs. Treatments were applied for 24 to 72 hours periods as indicated. For transfection experiments, cells were transfected with Lipofectamine 2000 (Invitrogen). Culture media were refreshed 18-24 $\mathrm{h}$ post-transfection, and cells were imaged $48 \mathrm{~h}$ post-transfection to allow sufficient recovery from transfection stress.

Plasmids and shRNAs. GFP-LC3 (\#21073), mCherry-Lamp1 (\#45147), and GFP-RFP-LC3 (\#84573) were obtained from addgene. mCherry-VAMP8 and GFP-Stx17 were kindly provided by Drs. Hong Zhang (National Institute of Biological Sciences, Beijing, China), Edward A Fon (Department of Neurology and Neurosurgery, McGill University), Amy Kiger (Department of Cell \& Developmental Biology, University of California, San Diego), and Qiming Sun (School of Basic Medical Sciences, Zhejiang University). NC (negative control) and TRPM7 shRNAs were designed and synthesized from GenePharma company. We chose the most efficient TRPM7 shRNA (labeled as T7 shRNA) out of 3 candidates to use in our experiments. The sequence of T7 shRNA was gcCAATATGTTCTACATTGTA. The ATG5-specific target sequence used was 5-AAGCAACTCTGGATGGGATTGC-3, corresponding to nucleotides 744-765 of the human ATG5 mRNA. shRNA T7 shRNA and ATG5 shRNA were then constructed into a Lentiviral vector for efficient knockdown of targeted TRPM7 and ATG5, respectively.

qPCR analysis. Total RNA from Patu 8988t, A-375, and U-87MG cells was isolated using RNAiso Trizol reagent (Sangon Biotech, China), and reverse-transcribed with PrimeScript ${ }^{\mathrm{TM}}$ RT Master Mix (Takara, Japan) according to the manufacturer's instructions. Then a TB GreenTM Premix EX TaqTM $\otimes$ (Takara, Japan) in a LightCycler 480 qPCR instrument (Roche) was used for qRT-PCR. qRT-PCR was performed in triplicate and the results were normalized against GAPDH. Relative fold expressions were calculated with the comparative threshold cycle $\left(2^{-\Delta \Delta C t}\right)$ method [52]. The sequences of all primers for qRT-PCR are listed in below:

hGAPDH-F: 5-AGCCACATCGCTCAGACAC-3

hGAPDH-R: 5-GCCCAATACGACCAAATCC-3

hTRPM7-F: 5'-CTTATGAAGAGGCAGGTCATGG-3'

hTRPM7-R: 5'-CATCTTGTCTGAAGGACTG-3' 
Electrophysiological recordings. Whole-cell patch-clamp recordings were carried out at room temperature. The external solution contained $145 \mathrm{mM} \mathrm{NaCl}, 5 \mathrm{mM} \mathrm{CsCl}, 2 \mathrm{mM} \mathrm{CaCl}_{2}, 1 \mathrm{mM} \mathrm{MgCl}_{2}, 20 \mathrm{mM} \mathrm{HEPES}$, and $10 \mathrm{mM}$ glucose ( $\mathrm{pH}$ adjusted to 7.4 with $\mathrm{NaOH}$ ). The pipette solution contained $120 \mathrm{mM} \mathrm{CsCl}, 10 \mathrm{mM}$ $\mathrm{NaCl}, 2 \mathrm{mM} \mathrm{MgCl} 2,1 \mathrm{mM}$ EGTA, and $10 \mathrm{mM} \mathrm{HEPES} \mathrm{(pH} 7.4$ adjusted with $\mathrm{CsOH}$ ). In some recordings, the pipette solution with $0 \mathrm{mM} \mathrm{Mg}^{2+}$ was prepared by removing $\mathrm{MgCl}_{2}$ and combining $5 \mathrm{mM}$ EDTA, as calculated with the Maxchelator program (http://maxchelator.stanford.edu/MgEDTA.htm). Data were acquired with an Axopatch 2A patch-clamp amplifier and a Digidata ${ }^{\circledR} 1440$ digitizer and recorded with pClamp 10.0 software (Axon Instruments). Whole-cell currents were digitized at $5 \mathrm{kHz}$ and filtered at 2 $\mathrm{kHz}$. All experiments were conducted at room temperature $\left(21-23^{\circ} \mathrm{C}\right)$ and all recordings were analyzed in pCLAMP10 (Axon Instruments) and Origin 8.0 (OriginLab) software.

Western blotting. Standard western blotting procedures were used. Antibodies used for Western blots include anti-LC3 (L8918, Sigma), anti-p62 (\#5114, Cell Signaling Technology), anti-S6K (\#9202, Cell Signaling Technology), anti-pS6K (\#9205, Cell Signaling Technology), anti-ULK1 (\#6439, Cell Signaling Technology), anti-pULK1 (\#14205, Cell Signaling Technology), anti-VAMP8 (\#13060, Cell Signaling Technology), anti-GAPDH (\#2118, Cell Signaling Technology), anti-ATG5 (\#2630, Cell Signaling Technology), p62 (\#GP62-C, Progen)and anti-GFP (ab290, Abcam).

Co-immunoprecipitation. Transfected cells from $10 \mathrm{~cm}$ dishes were incubated with $1 \mathrm{ml}$ of NP40 lysis buffer (1\% NP-40, $137.5 \mathrm{mM} \mathrm{NaCl}$, and 10\% Glycerol in $20 \mathrm{mM}$ Tris-HCl, adjusted to $\mathrm{pH} 7.4$ ) for $30 \mathrm{~min}$ at $4^{\circ} \mathrm{C}$. Lysates were centrifuged at $16,000 \mathrm{~g}$ for $10 \mathrm{~min}$, and supernatants were incubated with $1 \mathrm{mg}$ of primary antibodies at $4{ }^{\circ} \mathrm{C}$ overnight. Lysates were incubated at $4{ }^{\circ} \mathrm{C}$ for $1 \mathrm{~h}$ with gentle shaking following the addition of $20 \mathrm{ml}$ of protein $\mathrm{A} / \mathrm{G}$ plus agarose beads. Agarose beads were then collected through centrifugation at $500 \mathrm{~g}$ for $5 \mathrm{~min}$. Beads were washed three times thoroughly in lysis buffer, and then heated to $95^{\circ} \mathrm{C}$ for $5 \mathrm{~min}$ in NuPAGE loading buffer. Proteins were blotted with anti-GFP and anti-VAMP8 $(1: 500)$.

Immunofluorescence and immunohistology staining. Tumor tissues from mice were fixed with $4 \%$ paraformaldehyde, embedded with paraffin, and cut at $6 \mathrm{~mm}$. Histological sections were dewaxed, antigen retrieved with citric acid buffer, blocked with $3 \%$ hydroperoxide for 10 minutes, and then incubated with an antibody against p62 (1:300 progen) at $4^{\circ} \mathrm{C}$ overnight and then incubated with secondary antibody (Alexa Fluor 488 ) for $1.5 \mathrm{~h}$ at room temperature. The sections were counterstained with hematoxylin for $5 \mathrm{~min}$, dehydrated, cleared and mounted in neutral gum. TUNEL staining was performed according to the instructions (Roche). The tissue slices were counterstained with 4',6diamidino-2-phenylindole (DAPI, Sigma) for $3 \mathrm{~min}$. Diaminobenzidine was used as a chromogen to visualize the antibody/antigen complex. For immunohistochemistry experiments, tumor tissues were fixed and cryosectioned. Processed tissue sections were first incubated with ki67 antibody (\#9027, Cell Signaling Technology). Biotinylated secondary antibody was added, followed by the addition of preformed $A B C$ reagent (Santa Cruz). The target protein was visualized by incubation with peroxidase substrate. All sections were observed using the confocal laser scanning microscope. We counted 
percentage of cells positive for p62, TUNEL, and ki67 per field from different tumor sections from PBS and Clozapine groups, respectively ( $n=8-20$ sections). For p62 quantification, cells with overt puncta structures were counted for positive cells.

Trypan Blue assay. Freshly detached cells were diluted with same volume of trypan blue solution. $10 \mathrm{~mL}$ of sample was loaded into an hemacytometer and examined immediately under a microscope at low magnification. The number of blue staining cells and the number of total cells were counted. Cell viability was determined as [1.00 - (Number of blue cells/Number of total cells)].

Flow cytometry. For flow cytometry analysis, Patu 8988t cells were treated with different drugs as indicated in medium containing $2 \%$ FBS. Cells were collected, washed twice with PBS and stained using the Annexin V/7-AAD for apoptosis assay, H2DCFDA for ROS measurement, and Propidium lodide (PI) for cell cycle assay according to the manufacturer's instruction. Stained cells were analyzed with a BD FACSAria III flow cytometer and data was processed using FlowJo software.

Transwell migration assay. Cells were harvested and resuspended at $2 \times 10^{4}$ cells/well in serum-free DMEM medium. A total of $200 \mathrm{~mL}$ of the suspension was added into upper compartment of the transwell insert (\#3422, Corning), while a total of $600 \mathrm{ml}$ of complete DMEM medium containing $10 \%$ FBS was put into the lower compartment. After $16 \mathrm{~h}-24 \mathrm{~h}$ with or without drug treatments, migrated cells through microporous membrane of the insert were then fixed and stained by $0.5 \%$ crystal violet solution. Migrated cells were visualized under Olympus microscope for imaging and then eluted by $20 \%$ acetic acid for measuring OD600.

Transwell invasion assay. Matrigel (\#356234, BD Biosciences) was diluted by serum-free DMEM medium at 1:7. A total of $50 \mathrm{~mL}$ of the mixture was then used to coat transwell insert for $1-2 \mathrm{~h}$ at $370 \mathrm{C}$. Cells were harvested and resuspended at $5 \times 10^{4}$ cells/well in serum-free DMEM medium. A total of $200 \mathrm{~mL}$ of the suspension was added into upper compartment of the transwell, while a total of $600 \mathrm{ml}$ of complete DMEM medium containing 10\% FBS was put into the lower compartment. After $16 \mathrm{~h}-24 \mathrm{~h}$, invaded cells through the coated microporous membrane were then fixed and stained by $0.5 \%$ crystal violet solution (non-invasive cells removed from the top of the membrane with a cotton swab). Invaded cells were visualized under a microscope for imaging, and then eluted by $20 \%$ acetic acid for measuring OD600.

Mouse Xenografts. 1-5 X $10^{6}$ Patu $8988 \mathrm{t}$ cells were re-suspended in $50 \%$ Matrigel (BD Biosciences) to a final volume of $100 \mathrm{~mL}$ and then injected slowly into the subcutaneous tissue of $\sim 6$-week-old BALB/C nude mice. When the tumor diameter reached $10 \mathrm{~mm}$, PBS or Clozapine was daily administrated as indicated. Tumor volumes were measured daily and calculated by using the equation (length $\mathrm{X}$ width $\left.{ }^{2}\right) / 2$ for 14 days. Mice were sacrificed and tumors were collected for immunohistochemistry and immunoblotting experiments.

Melanoma lung-metastasis animal model. $0.5-1 \times 10^{6}$ cells were re-suspended to a final volume of 200 $\mathrm{mL}$ and then injected slowly into tails of $\sim 6$-week-old BALB/c nude mice. From the third day, PBS or 
Clozapine ( $3 \mathrm{mg} / \mathrm{kg}$ ) was intraperitonealy injected daily for 3 weeks. Mice were then sacrificed and lungs were collected for Hematoxylin and eosin (HE) staining.

Confocal imaging. Cells were grown on glass coverslips, fixed with 4\% PFA. After three washes with PBS, the coverslips were mounted with Fluoromount-G (Southern Biotech) and were processed for imaging using an Olympus confocal microscope.

DQ-BSA proteolytic assay. DQ-red-BSA was used as an artificial substrate to evaluate lysosomal proteolytic degradation (Wang et al., 2017; Yue et al., 2013). Briefly, cells were treated with DQ-red-BSA (10 $\mathrm{mg} / \mathrm{ml}$ ) for $2 \mathrm{~h}$ at $37^{\circ} \mathrm{C}$. After removal of extracellular DQ-red-BSA, cells were starved (amino acid free and serum withdrawal) to trigger autophagic degradation. Upon cleavage, DQ-red-BSA exhibited red fluorescence, as detected by confocal imaging.

Zinc staining. Hela cells were seeded on glass coverslips and loaded with $100 \mathrm{mM} \mathrm{ZnCl}_{2}$ for $12 \mathrm{~h}$ prior to applying different treatments indicated. Cells were washed 3 times with PBS, and then loaded with 2.5 mM FluoZin-3 AM for not less than 30 min. Confocal microscopy was performed using Olympus confocal microscopes.

\section{Reagents}

All reagents were dissolved and stored in DMSO or water. Baf-A1, Rapamycin, Torin-1, zVADfmk was obtained from Med Chem Express. DQ-BSA-red, FluoZin-3 AM, LysoTracker Red DND-99, BAPTA-am, EDTA, PI, and H2DCFDA were from Life Technologies. Clozapine, FTY720, NS8593, TTM, DIP, Annexin V/7-AAD, CQ, 3-MA, TPEN, 1,10 Phenanthroline were purchased from Sigma. Trypan blue was purchased from VWR. Naltriben was purchased from Santa Cruz Biotechnology.

Statistical Analysis. Data are presented as the means \pm standard errors of the mean (SEMs) from at least three independent experiments. Statistical significance of differences was evaluated using ANOVA followed by Tukey's test. $P$ values $<0.05$ were considered statistically significant.

\section{Results}

\section{Stimulating TRPM7 channel specifically inhibits autophagy.}

We selected 4 relatively potent compounds, Clozapine, Naltriben, FTY720, and NS8593, which have been well characterized ${ }^{[19,20,22]}$, to either stimulate or inhibit TRPM7 channel activity. We also confirmed the efficiency of these small molecules on manipulating endogenous TRPM7 channel activity in the cells that we used in the study including Hela, HEK 293T, Patu 8988 t and A-375 cells (Figure S1A, B, C, D). In these cells, we were able to record endogenous TRPM7 currents that are largely inhibited by internal $\mathrm{Mg}^{2+}(2$ $\mathrm{mM})$. Furthermore, treatment of Clozapine $(20 \mathrm{mM})$ or Naltriben $(5 \mathrm{mM})$ significantly increased TRPM7 currents that were completely abolished by the application of FTY $720(1 \mathrm{mM})$ or NS8593 $(20 \mathrm{mM})$. To explore the effects of TRPM7 on autophagy, we first measured changes in microtubule associated protein 
1 light chain 3 (LC3) and p62, which are critical molecules involved in autophagy ${ }^{[24]}$. Application of Rapamycin $(50 \mu \mathrm{M})$, a mTORC1 inhibitor known to activate the autophagy process ${ }^{[24]}$, led to an increase in LC3II levels and a decrease in p62 levels in Hela cells, which validated our western blot assay for accurately measuring the changes in LC3II and p62. Treatment with Clozapine $(20 \mu \mathrm{M})$ or Naltriben $(5$ $\mu \mathrm{M}$ ) resulted in a significant increase in both LC3II and p62 protein levels in Hela cells (Fig. 1A, B, C; treatments for 4 hours $(\mathrm{h})$ ). The effects on increasing LC3II levels are dependent on concentrations and treatment periods of Clozapine or Naltriben (Figure S1E and S1F). Furthermore, the increases in LC3II by Clozapine or Naltriben were not further facilitated by co-application of Bafilomycin-A1 (Baf-A1 $(1 \mu \mathrm{M})$; Fig. 1A, B), which arrests autophagy at late step by impairing lysosomal function ${ }^{[25]}$. By contrast, increased LC3II levels mediated by starvation (amino acid (AA) free and Fetal Bovine Serum (FBS) free) were further facilitated by Baf-A1 (1 $\mu \mathrm{M}$; Figure S1G). When taken together, these results together suggested that the treatment of Clozapine or Naltriben inhibits autophagy.

Consistent with the results obtained by pharmacologically manipulating TRPM7 activity, up-regulation of TRPM7 channels using lenti-TRPM7 overexpression (OE-TRPM7) increased LC3Il levels in Hela cells (Fig. 1D and S1H, I). In addition to this, OE-TRPM7 resulted in the formation of LC3 puncta structures which indicate autophagosomes accumulation in GFP-LC3 stably expressed HEK 293T cells (S-GFP-LC3) (Fig. 1E). p62 puncta structures were also increased in OE-TRPM7 Hela cells that were overexpressing RFP-p62 (Fig. 1F). As TRPM7 possesses both channel and kinase properties, we wanted to determine which of these functions was involved in TRPM7-regulation of autophagy. As such, we evaluated the effects of a truncated form of the TRPM7 protein consisting of amino acids 1-1537 (lacking the kinase domain) ${ }^{[26]}$. Overexpression of the truncated TRPM7 protein (OE-truncated-TRPM7) significantly increased LC3 and p62 puncta in GFP-LC3 or RFP-p62 transiently expressed Hela cells, and the increase in LC3 and p62 puncta was comparable to cells overexpressing the full length TRPM7 protein (Fig. 1G, F). These results indicate that the channel segment of TRPM7 is responsible for inhibiting autophagy.

Interestingly, down-regulation of TRPM7 with a TRPM7 shRNA (T7 shRNA) (Figure S1J) did not significantly alter LC3Il levels in Hela cells (Fig. 1G). This suggests that TRPM7 may not be involved in regulating basal autophagy. When taken together, these results demonstrate that stimulating TRPM7 channels either by using pharmacological agonists or by increasing the channel expression levels inhibits autophagy.

To exclude possible off-target effects of Clozapine or Naltriben, we used two cell lines, WT normal rat kidney epithelial (NRK) cells and the corresponding ATG12 KO NRK cells (Figure S1K), in which autophagy is not able to be initiated ${ }^{[27]}$. In WT NRK cells, treatment with Clozapine $(20 \mu \mathrm{M})$ or Naltriben (5 $\mu \mathrm{M})$ induced a robust LC3II increase, whereas Clozapine $(20 \mu \mathrm{M})$ or Naltriben $(5 \mu \mathrm{M})$ in ATG12 KO cells failed to trigger any detectable levels of LC3II (Fig. 2A). Similarly, when autophagy initiation was blocked by pre-treating cells with 3-Methyladenine (3-MA; $10 \mathrm{mM}$ for $2 \mathrm{~h}$ ), a Phosphatidylinositol 3-kinases (PI3K) inhibitor ${ }^{[24]}$, the effects of Clozapine $(20 \mu \mathrm{M})$ or Naltriben $(5 \mu \mathrm{M})$ on increasing LC3II levels and LC3 
puncta structures were largely compromised (Fig. 2B and Figure S1L). These experiments confirm the specific effects of Clozapine or Naltriben on regulating autophagy.

Next, we wanted to determine if the effect of Clozapine or Naltriben on autophagy is mediated by TRPM7 channels, we utilized two TRPM7 antagonists, FTY720 and NS8593 ${ }^{[20]}$, and TRPM7 shRNA (T7 shRNA) to abolish TRPM7 channel activity. First, we confirmed that treatment with Clozapine or Naltriben did not affect TRPM7 expression levels (Figure S2A). Next, LC3 puncta structures induced by Clozapine $(20 \mu \mathrm{M})$ or Naltriben $(5 \mu \mathrm{M})$ in S-GFP-LC3 cells were markedly reduced by co-application of either FTY720 $(1 \mu \mathrm{M})$ or NS8593 $(20 \mu \mathrm{M})$ (Fig. 2C and S1A-D), indicating that treatment with Clozapine or Naltriben regulates autophagy by modulating TRPM7 channel activity. Third, we utilized a T7 shRNA to efficiently reduce TRPM7 expression levels in several cell lines (Figure S1J). We found that the increases in LC3Il caused by Clozapine $(20 \mu \mathrm{M})$ or Naltriben $(5 \mu \mathrm{M})$ were profoundly abolished in Hela cells transduced with the T7 shRNA, while being remained in cells transduced with a negative control shRNA (NC shRNA; Fig. 1G), confirming that Clozapine or Naltriben regulates autophagy by modulating TRPM7 channel activity. Supporting this conclusion, in comparison with overt mCherry-LC3 puncta in mCherry-LC3 and GFP-NC shRNA transiently co-expressed Hela cells, Clozapine or Naltriben barely triggered mCherry-LC3 puncta in mCherry-LC3 and GFP-T7 shRNA transiently co-expressed Hela cells (Fig. 2D). When taken together, these results demonstrated that activation of TRPM7 specifically regulates (inhibits) autophagy.

\section{Activation of TRPM7 inhibits autophagy by disrupting fusion of autophagosomes and lysosomes.}

The LC3II and p62 western blot results indicate that activation of TRPM7 inhibits autophagy at the late step (Fig. 1A, B, C). As such, we wanted to systematically study the mechanism through which autophagic flux is inhibited by activation of TRPM7. First, we assessed autophagy initiation by measuring the activity of mammalian target of rapamycin complex 1 (mTORC1) and Unc-51 Like Autophagy Activating Kinase 1 (ULK1) ${ }^{[24]}$. As shown in Fig. 3A, application of Clozapine (20 $\mu \mathrm{M}$ for $12 \mathrm{~h}$ ) or Naltriben $(5 \mu \mathrm{M})$ did not alter levels of phosphorylated S6 Kinase (pS6K) or phosphorylated ULK1 (pULK1), which represents active mTORC1 and ULK1, respectively. In contrast, treatment with Torin-1 (1 $\mu \mathrm{M})$, a potent inhibitor of $\mathrm{mTORC1}$, markedly reduced both $\mathrm{PS} 6 \mathrm{~K}$ and PULK1 levels, thereby validating the assay for accurately assessing autophagy initiation. These results suggest that activation of TRPM7 does not influence autophagy initiation.

Next, we used a tandem plasmid GFP-RFP-LC3 to track autophagic flux. We observed significantly more red puncta (RFP-LC3) than green puncta (GFP-LC3) in GFP-RFP-LC3 transiently expressed Hela cells under starvation conditions (AA and FBS free; bottom panels; Fig. 3B). In contrast, Clozapine ( $20 \mu \mathrm{M}$ for $4 \mathrm{~h}$ ) or Naltriben $(5 \mu \mathrm{M})$ treatment induced equal numbers of red and green puncta (middle panels; Fig. 3B), suggesting that activation of TRPM7 inhibits autophagic flux at the late stage. These imaging results are consistent with the western blot results for LC3II and p62 (Fig. 1A, B, C). Additionally, we measured GFP turnover to evaluate autophagic flux following treatment with Clozapine $(20 \mu \mathrm{M})$ or Naltriben $(5 \mu \mathrm{M})$ in GFP-LC3 stably expressed HEK 293T cells (S-GFP-LC3) ${ }^{[28]}$. Treatment of Clozapine or Naltriben markedly impaired GFP turnover (Figure S2B) in the same way as Chloroquine (CQ) $(10 \mu \mathrm{M})$, which inhibits 
autophagy by eliminating lysosomal functions. Collectively, these results indicate that activation of TRPM7 inhibits autophagy at the late step.

To further define which process is inhibited by the activation of TRPM7 at late step of autophagy, we assessed the fusion process of autophagosomes and lysosomes as well as lysosomal functions following the activation of TRPM7. Neither Clozapine $(20 \mu \mathrm{M})$ nor Naltriben $(5 \mu \mathrm{M})$ altered lysosomal degradative capability (Fig. 3C), assessed using a DQ-BSA assay ${ }^{[29,30]}$, whereas starvation conditions significantly enhanced and Baf-A1 treatment reduced lysosomal functions and served as positive controls for the assay ${ }^{[30]}$. Furthermore, the lysosomal acidified environment was not changed by treatment with Clozapine $(20 \mu \mathrm{M})$ or Naltriben $(5 \mu \mathrm{M})$, as assessed using a LysoTracker probe which only stains acidic lysosomes (Figure S2C) ${ }^{[31]}$. This set of experiments confirms that Clozapine or Naltriben do not alter lysosomal functions and therefore eliminate the possibility that activation of TRPM7 inhibits autophagy by impairing lysosomal function.

We then tracked the fusion process between autophagosomes and lysosomes following treatment with Clozapine (20 $\mu \mathrm{M}$ for $4 \mathrm{~h})$ or Naltriben $(5 \mu \mathrm{M})$. In contrast to CQ treatments (bottom panels in Fig. 3D), most LC3 puncta ( 80\%; Fig. 3D) induced by Clozapine or Naltriben were adjacent to (arrows) and not fused to lysosomes stained with LysoTracker in GFP-LC3 transiently expressed Hela cells. This indicates that the fusion process between autophagosomes and lysosomes is repressed by TRPM7 activation. In addition, we confirmed the disturbed fusion between autophagosomes and lysosomes using an alternative approach that labels autophagosomes with GFP-LC3 and lysosomes with mCherry-Lamp1 (lysosomal associated membrane protein 1) (Figure S2D) in GFP-LC3 and mCherry-Lamp1 co-expressed Hela cells. When taken together, these results demonstrate that activation of TRPM7 disrupts the fusion between autophagosomes and lysosomes and subsequently inhibits autophagy.

\section{Activation of TRPM7 mediates zinc release to the cytosol, which in turn eliminates the interaction between Stx17 and VAMP8 and in turn inhibits fusion of autophagosomes and lysosomes.}

Given that the effects of Clozapine or Naltriben on increasing LC3II levels were observed within as early as $2 \mathrm{~h}$ of treatment (Figure S1F), we hypothesize that the effects of TRPM7 on autophagy were attributed to cationic flux through the channel. Accordingly, we used different chelators to define which cation is the downstream effector of TRPM7 governing autophagy. Considering the formulation of Dulbecco's Modified Eagle Medium (DMEM; Gibco) used in our experiments mainly contains $\mathrm{Ca}^{2+}(\sim 1.8 \mathrm{mM})$ and $\mathrm{Mg}^{2+}(\sim 0.8 \mathrm{mM})$ without zinc included, we used Ethylenediaminetetraacetic acid (EDTA, $5 \mathrm{mM}$; (http://web.stanford.edu/ cpatton/webmaxS.htm)) to completely chelate extracellular $\mathrm{Ca}^{2+}$ and $\mathrm{Mg}^{2+}$. However, EDTA application ( $5 \mathrm{mM})$ did not affect the Clozapine $(20 \mu \mathrm{M}$ for $4 \mathrm{~h})$ mediated increase in LC3II levels (Fig. 4A), indicating that intracellular cations flux through TRPM7 may play a role in regulating autophagy. A previous study from Dr. Clapham's group ${ }^{[7]}$ has suggested that majority of TRPM7 localizes in an unknown intracellular organelle that accumulates a large quantity of zinc. Therefore, we ask if the intracellular zinc release mediated by TRPM7 activation regulates autophagy. We initially confirmed that treatment with Clozapine or Naltriben led to a significant elevation in cytosolic 
zinc concentrations in Hela cells stained by a FluoZin-3 AM probe ${ }^{[32]}$ (Fig. 4B). Application of the zinc chelator N,N,N',N'-Tetrakis(2-pyridylmethyl)ethylenediamine (TPEN, $10 \mu \mathrm{M}$ ) completely chelated cytosolic elevated zinc concentrations that resulted from treatment with Clozapine $(20 \mu \mathrm{M}$ for $4 \mathrm{~h})$ or Naltriben (5 $\mu \mathrm{M})$ (Fig. 4B), thus validating the assay for accurately monitoring cytosolic zinc levels. Moreover, the increase in cytosolic zinc concentrations by Clozapine or Naltriben were markedly repressed by coapplication with NS8593 $(20 \mu \mathrm{M})$ or FTY720 $(1 \mu \mathrm{M})$ (Fig. 4B). This further suggests that Clozapine or Naltriben mediates zinc release to the cytosol by stimulating TRPM7. Surprisingly, application of TPEN $(10 \mu \mathrm{M})$ or 1,10 Phenanthroline $\left(1,10^{\prime}\right.$ Phe, $\left.500 \mu \mathrm{M}\right)$, two specific zinc chelators that have much higher affinity for zinc than $\mathrm{Ca}^{2+}$ or other cations at the concentrations we used ${ }^{[33]}$, profoundly rescued the increase in LC3II levels by Clozapine $(20 \mu \mathrm{M})$ (Fig. 4A). This indicates that the intracellular zinc release to the cytosol mediated by TRPM7 is the effector regulating autophagy. To further confirm this, we used $\mathrm{Fe}^{2+}$ chelator, tachpyr, ${ }^{[34]}, \mathrm{Ca}^{2+}$ chelator, 1,2-Bis(2-aminophenoxy)ethane-N,N,N',N'-tetraacetic acid tetrakis-acetoxymethyl ester (BAPTA-am) ${ }^{[35]}$, and $\mathrm{Cu}^{2+}$ chelator, tetrathiomolybdate (TTM) ${ }^{[36,37]}$ and none of these chelators reversed the increase in LC3II levels resulting from Clozapine treatment (Figure S2E). In addition to abolishing the effects of Clozapine, TPEN application (10 $\mu \mathrm{M})$ significantly rescued increased LC3II levels triggered by Naltriben application ( $5 \mu \mathrm{M}$; Fig. $4 \mathrm{C})$ and yielded greater effects on reversing the increase in LC3II levels with longer treatments for $12 \mathrm{~h}$ (vs $4 \mathrm{~h}$ in Fig. $4 \mathrm{~A}$ ). Consistent with the alterations in LC3II protein levels (Fig. 4A, C), TPEN application $(10 \mu \mathrm{M})$ markedly reduced the number of LC3 puncta induced by Clozapine or Naltriben treatment in GFP-LC3 stably expressed HEK 293T cells (Fig. 4D). Additionally, application of TPEN (10 mM) or 1,10 Phe $(500 \mathrm{mM})$ profoundly abolished the increases in LC3II levels by treatment with Clozapine $(20 \mathrm{mM})$ or Naltriben $(5 \mathrm{mM})$ in cancer cells including a human pancreatic cancer cell line (Patu 8988t) and a human melanoma cell line (A-375 cells) (Figure S2F, G). Collectively, these results demonstrate that activation of TRPM7 inhibits autophagy by mediating intracellular zinc release to the cytosol, presumably from the unknown zinc-storing vesicles ${ }^{[7]}$.

Next, we sought to identify what is the downstream effector(s) of zinc release mediated by TRPM7 that are disrupting the fusion between autophagosomes and lysosomes. As such, we examined the interaction between two SNARE proteins, syntaxin17 (Stx17) and VAMP8, as they determine fusion status between autophagosomes and lysosomes ${ }^{[23]}$. In contrast to starvation conditions (bottom panels; Fig. 4E), most of Stx17 puncta ( 85\%) triggered by Clozapine or Naltriben treatment were not colocalized with VAMP8 in Hela cells transiently co-expressing GFP-Stx17 and mCherry-VAMP8 (Fig. 4E, F). In addition to this, the physical interaction between Stx17 and VAMP8 was eliminated by application of Clozapine (20 $\mu \mathrm{M}$ for $12 \mathrm{~h}$ ) or Naltriben $(5 \mu \mathrm{M})$, as assessed by co-immunoprecipitation experiments (Fig. 4G, H). Using the approached outline in Figure $\mathbf{S 2 H}$, treatment with Clozapine $(20 \mu \mathrm{M})$ or Naltriben $(10 \mu \mathrm{M})$ significantly inhibited the interaction between Stx17 and VAMP8 whereas starvation conditions (AA and FBS free) facilitated the interaction ${ }^{[23]}$. To further investigate whether cytosolic zinc levels directly regulate the interaction between Stx17 and VAMP8, we applied TPEN (10 $\mu \mathrm{M})$ after the lysates have been collected. We found that TPEN strongly attenuated the eliminated interaction between Stx17 and VAMP8 by Clozapine or Naltriben treament (Fig. 4G, H), suggesting the release of zinc through TRPM7 channels directly affects the interaction between Stx17 and VAMP8. 
Overall, these results suggest that the activation of TRPM7 mediates intracellular zinc release to the cytosol, which in turn curbs the interaction between Stx17 in the autophagosome and VAMP8 in the lysosome and consequently inhibits autophagy.

\section{Stimulating TRPM7 channels triggers cell death in a variety of cancer cells with little effects on normal cells.}

Autophagy inhibition limits the nutrient supply for malignant cells and therefore is able to efficiently restrict the growth of cancer cells ${ }^{[13]}$. Although Hela cells were rather sensitive to TRPM7-mediated autophagy inhibition (Figure S3A), considering the molecular and phenotypic variability across Hela cells $^{[38]}$, instead of using Hela cells, we investigated the effects of TRPM7-mediated autophagy inhibition on viability of other types of cancer cells, including Patu 8988t (a human pancreatic cancer cell line; Figure S3B, C), MCF-7 (a human breast cancer cell line; Figure S3D and S3E), and A-375 (a human melanoma cell line; Figure S3F, G) cells as well as their corresponding normal cells. We first confirmed that treatment with Clozapine or Naltriben inhibits autophagy in each of these cancer cells that we used in the study such as Patu 8988t (Figure S3B, C), MCF-7 (Figure S3D, E) and A-375 (Figure S3F, G) cells to the same extent as Hela and HEK 293T, which was confirmed by measuring changes in LC3II and p62 levels. When challenged with starvations, cancer cells are extremely sensitive to autophagy inhibition, we therefore examined the effects of Clozapine or Naltriben on cancer cells viability in DMEM medium with $2 \%$ FBS (data from $1 \%$ and $5 \%$ not shown) as well as on the corresponding normal cell lines for each cancer cell line. Treatment with Clozapine $(10,20,40 \mu \mathrm{M})$ or Naltriben $(5,10 \mu \mathrm{M})$ for different periods profoundly induced overt cell death in Patu 8988t (Fig. 5A, B), MCF-7 (Fig. 5C, D), SGC-7901 (a human gastric cancer cell line; Fig. 5E, F), A-375 (Fig. 5G) cells, and U-87 MG (a human glioma cell line; Fig. 5H) cells as assessed using a Trypan blue assay. Notably, much weaker or negligible effects were observed on the viability of the corresponding normal cell lines including HPDE6c7 (a human pancreatic ductal epithelial cell line; Fig. 5A, B), MCF-10A (a human breast gland epithelial cell; Fig. 5C, D), and GES-1 (a human gastric mucosal epithelial cell line; Fig. 5E, F) following treatment with Clozapine or Naltriben. We reasoned that autophagy in normal cells is not as active as in cancer cells to survive limited nutrients, which was supported by the observation that the corresponding normal cells exhibited much lower basal autophagy levels than the cancer cells (Figure S3H). In other words, unlike cancer cells, normal cells do not highly depend on autophagy for growth. Thus, negligible effects of Clozapine or Naltriben on viability of normal cells were observed.

Collectively, autophagy inhibition by Clozapine or Naltriben induces cell death in an array of cancer cells. More importantly, the feature of Clozapine or Naltriben on triggering cancer cell death with limited effects on normal cells will evidently broaden their therapeutic potential to treat cancer (see discussions).

\section{Autophagy inhibition mediated by TRPM7 suppresses cancer cells metastasis.}

Autophagy regulates metastatic progression by modulating multiple steps in the metastatic cascade ${ }^{[11}$,

39]. Therefore, we investigated the effects of autophagy inhibition mediated by TRPM7 on cancer 
metastasis. We found that both migrated and invaded cancer cells including A-375 cells (Fig. 6A, B, C, D), Patu 8988t cells (Figure S4), MCF7 cells (Figure S5A, B), SGC-7901 cells (Figure S5C, D), and U-87 MG cells (Figure S5E, F), were robustly suppressed by treatment with Clozapine $(20 \mu \mathrm{M})$ or Naltriben $(10 \mu \mathrm{M})$, as assessed using Transwell assays (see details in methods). Moreover, pretreatment with 3-MA (10 mM for 2 h; Fig. 2B) significantly rescued both the suppressed migration and invasion initiated by Clozapine or Naltriben in A-375 and Patu 8988t cells (Fig. 6A, B, C, D and Figure S4). Similarly, knockdown of ATG5 significantly abolished the effects of Clozapine or Naltriben on suppressing metastasis in A-375 cells (Fig. 6E, F) and MCF-7 cells (Figure S5G, H), suggesting that autophagy inhibition resulting from Clozapine or Naltriben treatment specifically regulates metastasis in these cancer cell lines. Furthermore, knockdown of TRPM7 or co-application of FTY720 $(1 \mu \mathrm{M})$ largely rescued the suppressed metastasis observed in A-375 cells and Patu 8988t cells following Clozapine or Naltriben treatment. In contrast, application of FTY720-phosphate (FTY720-P), which has no effect on TRPM7 ${ }^{[40]}$, did not alter the suppression of migration and invasion elicited by treatment of Clozapine or Naltriben (Fig. 6A, B, C, D, G, $\mathrm{H}$ and Figure S4). This further confirms that the suppression of migration and invasion are mediated by TRPM7 channel activity. All together, these results indicate that autophagy inhibition mediated by TRPM7 robustly suppresses metastasis in the cancer cells.

\section{Stimulating TRPM7 with Clozapine restricts tumor growth and metastasis in vivo.}

So far, we have shown the effects of autophagy inhibition mediated by TRPM7 on suppressing cancer cells growth and metastasis in vitro. Next, we examined the effects of autophagy inhibition by stimulating in vivo TRPM7 using Clozapine, an atypical antipsychotic medication. We used the Patu 8988t Xenografts mice model and subjected mice to daily intragastric (i.g.) administration of Clozapine $(0.6 \mathrm{mg} / \mathrm{kg}$ and $2 \mathrm{mg} / \mathrm{kg}$ ) and observed a dramatic suppression in tumor growth in Xenografted mice (Fig. 7A, B). After 14 days of Clozapine administration (i.g.), the final tumor volume of the Clozapine group was reduced to less than half the size of tumors in the control group that received daily phosphate buffered saline (PBS) injections (Fig. 7A, B). Intratumoral injection of Clozapine (40 $\mu \mathrm{M})$ also yielded significant repressive effects on tumor growth (Fig. 7C, D). Co-injection of FTY720 (10 $\mu \mathrm{M})$ markedly compromised the repression on tumor growth mediated by Clozapine (Fig. 7C, D), suggestive of a specific role for TRPM7 on regulating tumor growth. These in vivo anti-neoplastic effects of Clozapine presumably result from the inhibition of autophagy regulated by TPPM7, as we observed that strong autophagy inhibition was induced by administration of Clozapine, which was supported by the observation that Clozapine treated mice exhibited a significant increase in the abundance of p62 puncta structures in excised tumor tissue samples compared to the PBS control (Fig. 7E, F). In addition, tumor biopsies from the Clozapine group exhibited an increase in terminal deoxynucleotidyl transferase dUTP nick end labeling (TUNEL) signal and reduced ki67 levels (Fig. 7E, F). When taken together, these in vivo results demonstrate that Clozapine has therapeutic potential for suppressing tumor growth by inhibiting autophagy through TRPM7.

Clozapine not only exhibited great effects on suppressing tumor growth, but also prevented metastasis. After 3 days of establishing a melanoma mouse model by Tail vein injecting A-375 cells, we 
intraperitoneally injected Clozapine daily for 3 weeks. In comparison with the PBS group, the incidence of melanoma in the lungs was profoundly reduced by Clozapine administration $(3 \mathrm{mg} / \mathrm{kg}$; doses lower than $3 \mathrm{mg} / \mathrm{kg}$ did not yield repressive effects on metastasis) (Fig. 7G), supported by immunohistochemical staining (Fig. 7H).

Taken together, inhibiting autophagy by stimulating TRPM7 with the administration of Clozapine exhibited great potentials for the suppression of tumor growth and metastasis in vivo, thus representing TRPM7 a therapeutic target to combat cancer.

\section{TRPM7-mediated autophagy inhibition triggers cell death by evoking apoptosis, cell cycle arrest, and ROS elevation.}

We next investigated how autophagy inhibition mediated by Clozapine or Naltriben treatment triggers cell death in the aforementioned cancer cells. Considering the interplay between autophagy and apoptosis ${ }^{[41]}$, we evaluated the extent of apoptosis using the same Clozapine or Naltriben treatment used in cell viability experiments ( $2 \% \mathrm{FBS})$. Following the application of Clozapine $(20 \mu \mathrm{M})$ or Naltriben $(5 \mu \mathrm{M})$ for 48 h, both early and late apoptosis was significantly induced in Patu 8988t cells as assessed by 7AAD/Annexin V levels (Fig. 8A and S6A-B). Furthermore, co-application of FTY720 (1 $\mu \mathrm{M})$ or knocking down TRPM7 with T7 shRNA (Figure S1J) profoundly reduced the extent of apoptosis triggered by treatment of Clozapine or Naltriben (Fig. 8A), whereas the application of FTY720 or T7 shRNA alone did not result in cell apoptosis compared to the control (Fig. 8A and Figure S6C). Together, these results together confirm that activation of TRPM7 induces apoptosis. Next, we investigated the changes in cell cycle of Patu 8988t cells following treatment with Clozapine or Naltriben using flow cytometry assays. Both Clozapine $(20 \mu \mathrm{M})$ and Naltriben $(5 \mu \mathrm{M})$ treatment arrested the cell cycle at G0/G1 phases (Fig. 8B). Finally, we measured intracellular ROS levels following Clozapine or Naltriben treatment in Patu 8988t cells. Following treatment with Clozapine $(20 \mu \mathrm{M}$ for $48 \mathrm{~h}$ ) or Naltriben ( $5 \mu \mathrm{M}$ for $48 \mathrm{~h})$, Ros levels were greatly elevated compared to the control group (Fig. 8C). Moreover, the elevation in ROS resulting from Clozapine or Naltriben was profoundly abrogated by application of FTY720 or the T7 shRNA (Fig. 8C and Figure S6D). This further demonstrates that activation of TRPM7 by Clozapine or Naltriben elevates ROS levels.

Interestingly, when exposing Patu 8988 t cells to Clozapine $(20 \mu \mathrm{M}$ for $48 \mathrm{~h})$ or Naltriben ( $5 \mu \mathrm{M})$ in medium containing $10 \%$ FBS, we observed that apoptosis, cell cycle arrest and ROS elevation did not occur in the cells (Figure S6E, F, G). These results could explain why Clozapine and Naltriben had little effects on normal cells (Fig. 5), in which nutrients are not limited.

Next, we asked whether changes in these cellular responses induced by activation of TRPM7 lead to cell death in the aforementioned cancer cell lines. Initially, we confirmed that cell death triggered by the treatment of Clozapine or Naltriben were mediated by TRPM7 through using T7 shRNA or TRPM7 antagonists. We observed that Clozapine $(20 \mu \mathrm{M} ; 48 \mathrm{~h})$ or Naltriben $(5 \mu \mathrm{M} ; 48 \mathrm{~h})$ treatment triggered overt cell death in Patu 8988t cells transduced with the NC shRNA (Fig. 8D). In contrast, neither Clozapine nor 
Naltriben caused significant cell death in cells transfected with T7 shRNA (Fig. 8D). Moreover, the coapplication of either FTY720 $(1 \mu \mathrm{M})$ or NS8593 $(20 \mu \mathrm{M})$ profoundly abolished cell death caused by Clozapine or Naltriben treatment in Patu 8988t cells (Fig. 8E, F). These results indicate that treatment of Clozapine or Naltriben results in cancer cell death by modulating TRPM7 activity. In order to confirm that TRPM7-mediated autophagy inhibition specifically leads to cell death in Patu 8988t cells, we pretreated Patu 8988 t cells with 3-MA ( $10 \mathrm{mM}$ for $2 \mathrm{~h}$; Fig. 2B) to block autophagy initiation. Following pretreatment with 3-MA, application of Clozapine $(20 \mu \mathrm{M}$ for $48 \mathrm{~h})$ or Naltriben $(5 \mu \mathrm{M}$ for $48 \mathrm{~h})$ did not cause significant cell death (Fig. 8E, F), suggesting autophagy inhibition specifically leads to cell death. In addition to this, a lentiviral-ATG5 shRNA was used to block autophagy initiation in Hela cells (Figure S6H), Patu 8988t cells (Figure S6I), MCF-7 cells (Figure S6I), and A375 cells (Figure S6I). Similar to the results using 3-MA, when autophagy initiation is blocked, the later application of Clozapine or Naltriben failed to lead to significant cell death in Patu 8988t cells (Fig. 8G), A-375 cells (Figure S6J), and MCF-7 cells (Figure S6K). These results demonstrate that autophagy inhibition mediated by TRPM7 activation is upstream of cell death. Third, to confirm the role of zinc on Clozapine or Naltriben-mediated cell death, we combined the zinc chelator TPEN with Clozapine or Naltriben treatment. Application of TPEN $(1 \mu \mathrm{M})$ completely abolished cell death in Patu 8988t cells induced by Clozapine or Naltriben treatment (Fig. 8E, F), confirming that zinc-mediated autophagy inhibition is the trigger for cell death in Patu 8988t cells following TRPM7 activation. Last, we used zVADfmk $(20 \mu \mathrm{M})$, an apoptosis inhibitor, and N-Acetyl-Lcysteine (NAC; $5 \mathrm{mM}$ ), an antioxidant reagent, to manipulate the corresponding cellular responses, respectively. We found that cell death induced by Clozapine or Naltriben treatment were rescued by application of either zVADfmk or NAC (Fig. 8E, F), thereby demonstrating that downstream events following autophagy inhibition mediated by TRPM7 activation, induction of apoptosis and ROS elevations, are the direct triggers for cell death in Patu 8988t cells.

When taken together, we determined that TRPM7-mediated autophagy inhibition triggers cell death in cancer cells by evoking apoptosis, cell cycle arrest, and ROS elevation.

\section{Discussion}

In this study, we systematically explored the effects of TRPM7 on regulating autophagy and the influence resulting from autophagy inhibition mediated by TRPM7 on cancer growth and metastasis. We demonstrated that activation of TRPM7 results in the release of zinc into the cytosol, which subsequently eliminates the interaction between Sxt17 in the autophagosome and VAMP8 in the lysosome. The consequence of disruption on the interaction between these two SNARE proteins by activation of TRPM7 is the arrested autophagy during the step in which autophagosomes and lysosomes fuse. More importantly, TRPM7-mediated autophagy inhibition suppresses tumor growth and metastasis both in vitro and in vivo by evoking apoptosis, cell cycle arrest, and ROS level elevation (Fig. 8H).

Even though the effects of autophagy inhibition on tumorigenesis are still under debate ${ }^{[13,39,42]}$, especially the effects on metastasis, we believe that our results provide further evidence that autophagy inhibition favors suppressing tumor growth and metastasis. The long-time disputes on promoting or 
suppressing tumorigenesis by autophagy inhibition are, in part, due to lack of specific autophagy inhibitors. To date, autophagy inhibitors including CQ, hydroxychloroquine (HCQ), and Baf-A1 have been commonly used in many studies to investigate the relationship between autophagy inhibition and cancer. However, these compounds do not have clear targets for inhibiting autophagy. Moreover, their antineoplastic effects largely stem from the modulation of pathways other than autophagy inhibition per $s e^{[43,44]}$. These flaws limit the ability of scientists to draw clear conclusions on how autophagy inhibition influences cancer.

On the one hand, TRPM7 expression has been aberrantly upregulated in certain cancer cell lines and patient tumor tissues such as human pancreatic adenocarcinoma, breast cancer, and Glioblastoma, suggesting a proliferative role of TRPM $7^{[45-47]}$. Supporting this theory, several non-specific antagonists of TRPM7 including Midazolam, Ginsenoside Rg3 ( $\mathrm{IC}_{50}$ of $\left.350 \mu \mathrm{M}\right)$, Ginsenoside Rd (IC 50 of $\left.350 \mu \mathrm{M}\right)$, Carvacrol $\left(\mathrm{IC}_{50}\right.$ of $\left.561 \mu \mathrm{M}\right)$ and Xyloketal B $\left(\mathrm{IC}_{50} \text { of } 287 \mu \mathrm{M}\right)^{[45]}$ have been reported to arrest malignant cells proliferation. However, effective concentrations of these antagonists $(250 \sim 600 \mu \mathrm{M})$ for inhibiting cancer cell proliferation are far beyond their concentrations for inhibiting TRPM7 channel activity (more than 10 fold higher), which makes it challenging to determine whether the effects of these antagonists on inhibiting cancer cells growth stem from manipulating TRPM7 or not.

On the other hand, one study reported that overexpression of TRPM7 largely impairs cell proliferation in HEK 293 cells $^{[5]}$, which supports our theory that stimulating TRPM7 suppresses tumor cell growth. Another study reported that short interfering RNA (siRNA) of TRPM7 rescues neuron cell death challenged by oxygen-glucose deprivation ${ }^{[18]}$. This is another relevant study to ours, in which starvation conditions were employed, and demonstrates that starvation-induced neuronal cell death is significantly rescued by knocking down TRPM7, which is highly consistent with our results using cancer cells. More recently, a study suggested that silencing TRPM7 promotes the growth of vascular endothelial cells via the extracellular signal-regulated kinase (ERK) pathway ${ }^{[16]}$, indicating that loss of TRPM7 expression does not inhibit but rather promotes cell proliferation which is in accordance with our current study.

Furthermore, a study reported that Clozapine induces autophagic cell death in non-small cell lung cancer cells $^{[48]}$. Although the study did not tackle the mechanisms through which Clozapine induces autophagic cell death, their results are consistent with our observations made in cell viability experiments following Clozapine treatment.

Our work provides a new target, TRPM7, for specifically inhibiting autophagy. Targeting at TRPM7, the treatment of both Clozapine and Naltriben suppresses malignant cells growth and metastasis by inducing autophagy inhibition. More importantly, Clozapine and Naltriben possess potent anti-neoplastic effects with limited effects on normal cells, therefore conferring TRPM7 great therapeutic potentials for treating cancer in the clinical practice. In particular, Clozapine is an FDA-approved anti-psychotic medication that has been implicated in the reduced occurrence of lung cancer in patients with schizophrenia ${ }^{[49-51]}$. Although the mechanisms responsible for the reduced incidence of lung cancer in these patients administrated with Clozapine are still unknown, several studies have demonstrated 
Clozapine's antineoplastic effect is owing to a strong cytotoxic effect. Based on our own results, the administration of Clozapine may trigger apoptosis in cancer cells by inhibiting autophagy through TRPM7. In addition to Clozapine and Naltriben, there have been several compounds identified as agonists of TRPM7 including Proadifen, Doxepin, Mibefradil, and U-73343 ${ }^{[19]}$. Unfortunately, these compounds lack specificity and potency for the clinical purpose. Developments of selective and novel TRPM7 agonists are therefore in high demand for the purpose of inhibiting autophagy and combating cancer.

Nevertheless, despite unsettled functions of TRPM7 on cell proliferation from previous studies, our work establishes a new role of TRPM7 in regulating tumorigenesis by modulating autophagy. More importantly, the feature of stimulating TRPM7 triggering cancer cells death with limited effects on normal cells broadens the potential of applying TRPM7 activators to cancer therapy.

\section{Conclusions}

We demonstrate that stimulating TRPM7 channels inhibits autophagy by disrupting the fusion between autophagosomes and lysosomes. Moreover, the TRPM7-induced autophagy inhibition suppresses cancer cell proliferation and metastasis by inducing apoptosis and cell cycle arrest. Our findings represent TRPM7 channels a great potential for cancer treatment.

\section{Abbreviations}

\section{TRPM7}

transient receptor potential melastatin-subfamily member 7; ROS:reactive oxygen species; Zinc:Zn2+; PI(4,5)P2:Phosphatidylinositol 4,5-bisphosphate; SNARE:Soluble N-Ethylmaleimide-Sensitive Factor (NSF) Attachment Protein Receptor; Stx17:syntaxin 17; VAMP8:Vesicle-associated membrane protein 8; Baf-A1:Bafilomycin-A1; LC3:microtubule associated protein 1 light chain 3; p62/SQSTM1:sequestosome 1; AA:amino acid; FBS:fetal bovine serum; HEK:human embryonic kidney; NRK:normal rat kidney epithelial cells; GAPDH:glyceraldehyde-3-phosphate dehydrogenase; ATG12:autophagy related 12; 3-MA:3Methyladenine; PI3K:Phosphatidylinositol 3-kinases; NC:negative control; mTORC1:mammalian target of rapamycin complex 1; ULK1:Unc-51 Like Autophagy Activating Kinase 1; S6K:ribosomal protein S6 kinase; CQ:Chloroquine; Lamp1:lysosomal associated membrane protein 1; DMEM:Dulbecco's Modified Eagle Medium; EDTA:Ethylenediaminetetraacetic acid; TPEN:N,N,N',N'-tetrakis(2pyridylmethyl)ethylenediamine; TTM:tetrathiomolybdate; BAPTA-am:1,2-Bis(2-aminophenoxy)ethane$\mathrm{N}, \mathrm{N}, \mathrm{N}^{\prime}, \mathrm{N}^{\prime}$-tetraacetic acid tetrakis-acetoxymethyl ester; NAC:N-Acetyl-L-cysteine; i.g.:intragastric; TUNEL:terminal deoxynucleotidyl transferase dUTP nick end labeling; PBS:phosphate buffered saline; HCQ:hydroxychloroquine; siRNA:short interfering RNA; shRNA:short hairpin RNA; ERK:extracellular signalregulated kinase.

\section{Declarations}


Ethics approval and consent to participate: Experiments on mice were performed in accordance with Xuzhou Medical University Animal Facility and Use Committee-approved protocols.

Consent for publication: Not applicable.

Availability of data and material: The data that support the findings of this study are available upon reasonable request.

Competing interests: The authors disclose no competing interests.

Funding: This work was supported by National Natural Science Foundation of China (NSFC) grants (81772559 to W. W; 81600967 to C. L; 81971212 to F. G), NSF grants of the Jiangsu Province (BK20170262 to W. W), Key University Science Research Project of Jiangsu Province (20KJA310001 to W.W), Jiangsu Specially-Appointed Professor award to W.W, Jiangsu Province Innovative and Entrepreneurial Talent program to W.W and Jiangsu Province Innovative and Entrepreneurial Team program to W.W.

Author contributions: JQ and WW conceived of the presented idea. YX, XW, MMW, YL, YHF, YZ, YJ, ZS, CL, $P Z, R C, M W, N L, L D$ and FG performed experiments and contributed to the interpretation of the results. FG, JQ and WW supervised the findings of this work. JQ and WW wrote the manuscript. All authors discussed the results and contributed to the final manuscript.

Acknowledgements: We are grateful to Drs. Li Yu (School of Life Sciences, Tsinghua University), Hong Zhang (National Institute of Biological Sciences, Beijing, China), Edward A Fon (Department of Neurology and Neurosurgery, McGill University), Amy Kiger (Department of Cell \& Developmental Biology, University of California, San Diego), Qiming Sun (School of Basic Medical Sciences, Zhejiang University) and Ling Chen (Guangzhou institutes of biomedicine and health, Chinese academy of sciences) for sharing the plasmids and cell lines. We are grateful to Dr. Hailey Jansen (Libin Cardiovascular Institute, University of Calgary, Canada) for critical reading through the manuscript and appreciate the encouragement and helpful comments from other members of the Wang laboratory.

\section{References}

1. Krapivinsky G, Krapivinsky L, Manasian Y, Clapham DE. The TRPM7 chanzyme is cleaved to release a chromatin-modifying kinase. Cell. 2014;157:1061-72.

2. Jin J, Desai BN, Navarro B, Donovan A, Andrews NC, Clapham DE. Deletion of Trpm7 disrupts embryonic development and thymopoiesis without altering Mg2 + homeostasis. Science. 2008;322:756-60.

3. Jin J, Wu LJ, Jun J, Cheng X, Xu H, Andrews NC, et al. The channel kinase, TRPM7, is required for early embryonic development. Proc Natl Acad Sci U S A. 2012;109:E225-33.

4. Runnels LW, Yue L, Clapham DE. The TRPM7 channel is inactivated by PIP(2) hydrolysis. Nat Cell Biol. 2002;4:329-36. 
5. Nadler MJ, Hermosura MC, Inabe K, Perraud AL, Zhu Q, Stokes AJ, et al. LTRPC7 is a Mg.ATPregulated divalent cation channel required for cell viability. Nature. 2001;411:590-5.

6. Chokshi R, Matsushita M, Kozak JA. Detailed examination of Mg2 + and pH sensitivity of human TRPM7 channels. Am J Physiol Cell Physiol. 2012;302:C1004-11.

7. Abiria SA, Krapivinsky G, Sah R, Santa-Cruz AG, Chaudhuri D, Zhang J, et al. TRPM7 senses oxidative stress to release $\mathrm{Zn}(2+)$ from unique intracellular vesicles. Proc Natl Acad Sci U S A. 2017;114:E6079-E88.

8. Colvin RA, Holmes WR, Fontaine CP, Maret W. Cytosolic zinc buffering and muffling: their role in intracellular zinc homeostasis. Metallomics. 2010;2:306-17.

9. Mizushima N, Levine B, Cuervo AM, Klionsky DJ. Autophagy fights disease through cellular selfdigestion. Nature. 2008;451:1069-75.

10. Klionsky DJ, Abdel-Aziz AK, Abdelfatah S, Abdellatif M, Abdoli A, Abel S, et al. Guidelines for the use and interpretation of assays for monitoring autophagy (4th edition). Autophagy 2021: 1-382.

11. Mowers EE, Sharifi MN, Macleod KF. Autophagy in cancer metastasis Oncogene. 2017;36:1619-30.

12. Galluzzi L, Bravo-San Pedro JM, Demaria S, Formenti SC, Kroemer G. Activating autophagy to potentiate immunogenic chemotherapy and radiation therapy. Nat Rev Clin Oncol. 2017;14:247-58.

13. White E. The role for autophagy in cancer. J Clin Invest. 2015;125:42-6.

14. White E, Mehnert JM, Chan CS. Autophagy, Metabolism, and Cancer. Clin Cancer Res. 2015;21:503746.

15. Oh HG, Chun YS, Park CS, Kim TW, Park MK, Chung S. Regulation of basal autophagy by transient receptor potential melastatin 7 (TRPM7) channel. Biochem Biophys Res Commun. 2015;463:7-12.

16. Inoue K, Xiong ZG. Silencing TRPM7 promotes growth/proliferation and nitric oxide production of vascular endothelial cells via the ERK pathway. Cardiovasc Res. 2009;83:547-57.

17. Hanano T, Hara Y, Shi J, Morita H, Umebayashi C, Mori E, et al. Involvement of TRPM7 in cell growth as a spontaneously activated $\mathrm{Ca} 2+$ entry pathway in human retinoblastoma cells. J Pharmacol Sci. 2004;95:403-19.

18. Aarts M, lihara K, Wei WL, Xiong ZG, Arundine M, Cerwinski W, et al. A key role for TRPM7 channels in anoxic neuronal death. Cell. 2003;115:863-77.

19. Hofmann T, Schafer S, Linseisen M, Sytik L, Gudermann T, Chubanov V. Activation of TRPM7 channels by small molecules under physiological conditions. Pflugers Arch. 2014;466:2177-89.

20. Chubanov V, Schafer S, Ferioli S, Gudermann T. Natural and Synthetic Modulators of the TRPM7 Channel. Cells. 2014;3:1089-101.

21. Chubanov V, Gudermann T. Mapping TRPM7 Function by NS8593. Int J Mol Sci 2020; 21.

22. Chubanov V, Ferioli S, Gudermann T. Assessment of TRPM7 functions by drug-like small molecules. Cell Calcium. 2017;67:166-73.

23. Itakura E, Kishi-Itakura C, Mizushima N. The hairpin-type tail-anchored SNARE syntaxin 17 targets to autophagosomes for fusion with endosomes/lysosomes. Cell. 2012;151:1256-69. 
24. !!!. INVALID CITATION !!!.

25. Yamamoto A, Tagawa Y, Yoshimori T, Moriyama Y, Masaki R, Tashiro Y. Bafilomycin A1 prevents maturation of autophagic vacuoles by inhibiting fusion between autophagosomes and lysosomes in rat hepatoma cell line, H-4-II-E cells. Cell Struct Funct. 1998;23:33-42.

26. Nadolni W, Zierler S. The Channel-Kinase TRPM7 as Novel Regulator of Immune System Homeostasis. Cells 2018; 7.

27. Malhotra R, Warne JP, Salas E, Xu AW, Debnath J. Loss of Atg12, but not Atg5, in proopiomelanocortin neurons exacerbates diet-induced obesity. Autophagy. 2015;11:145-54.

28. Ni HM, Bockus A, Wozniak AL, Jones K, Weinman S, Yin XM, et al. Dissecting the dynamic turnover of GFP-LC3 in the autolysosome. Autophagy. 2011;7:188-204.

29. Yue W, Hamai A, Tonelli G, Bauvy C, Nicolas V, Tharinger H, et al. Inhibition of the autophagic flux by salinomycin in breast cancer stem-like/progenitor cells interferes with their maintenance. Autophagy. 2013;9:714-29.

30. Wang W, Gao Q, Yang M, Zhang X, Yu L, Lawas M, et al. Up-regulation of lysosomal TRPML1 channels is essential for lysosomal adaptation to nutrient starvation. Proc Natl Acad Sci U S A. 2015;112:E1373-81.

31. Chen X, Bi Y, Wang T, Li P, Yan X, Hou S, et al. Lysosomal targeting with stable and sensitive fluorescent probes (Superior LysoProbes): applications for lysosome labeling and tracking during apoptosis. Sci Rep. 2015;5:9004.

32. Kukic I, Lee JK, Coblentz J, Kelleher SL, Kiselyov K. Zinc-dependent lysosomal enlargement in TRPML1-deficient cells involves MTF-1 transcription factor and ZnT4 (Slc30a4) transporter. Biochem J. 2013;451:155-63.

33. Radford RJ, Lippard SJ. Chelators for investigating zinc metalloneurochemistry. Curr Opin Chem Biol. 2013;17:129-36.

34. Heath JL, Weiss JM, Lavau CP, Wechsler DS. Iron deprivation in cancer-potential therapeutic implications. Nutrients. 2013;5:2836-59.

35. Wang W, Zhang X, Gao Q, Lawas M, Yu L, Cheng X, et al. A voltage-dependent K(+) channel in the lysosome is required for refilling lysosomal Ca(2+) stores. J Cell Biol. 2017;216:1715-30.

36. Smirnova J, Kabin E, Jarving I, Bragina O, Tougu V, Plitz T, et al. Copper(I)-binding properties of decoppering drugs for the treatment of Wilson disease. alpha-Lipoic acid as a potential anti-copper agent. Sci Rep. 2018;8:1463.

37. Czlonkowska A, Litwin T, Dusek P, Ferenci P, Lutsenko S, Medici V, et al. Wilson disease. Nat Rev Dis Primers. 2018;4:21.

38. Liu Y, Mi Y, Mueller T, Kreibich S, Williams EG, Van Drogen A, et al. Multi-omic measurements of heterogeneity in HeLa cells across laboratories. Nat Biotechnol. 2019;37:314-22.

39. Dower CM, Wills CA, Frisch SM, Wang HG. Mechanisms and context underlying the role of autophagy in cancer metastasis. Autophagy. 2018;14:1110-28. 
40. Qin X, Yue Z, Sun B, Yang W, Xie J, Ni E, et al. Sphingosine and FTY720 are potent inhibitors of the transient receptor potential melastatin 7 (TRPM7) channels. Br J Pharmacol. 2013;168:1294-312.

41. Marino G, Niso-Santano M, Baehrecke EH, Kroemer G. Self-consumption: the interplay of autophagy and apoptosis. Nat Rev Mol Cell Biol. 2014;15:81-94.

42. Mathew R, Karantza-Wadsworth V, White E. Role of autophagy in cancer. Nat Rev Cancer. 2007;7:961-7.

43. Maes H, Kuchnio A, Peric A, Moens S, Nys K, De Bock K, et al. Tumor vessel normalization by chloroquine independent of autophagy. Cancer Cell. 2014;26:190-206.

44. Maycotte P, Aryal S, Cummings CT, Thorburn J, Morgan MJ, Thorburn A. Chloroquine sensitizes breast cancer cells to chemotherapy independent of autophagy. Autophagy. 2012;8:200-12.

45. Yee NS. Role of TRPM7 in Cancer: Potential as Molecular Biomarker and Therapeutic Target. Pharmaceuticals (Basel) 2017; 10.

46. Gautier M, Perriere M, Monet M, Vanlaeys A, Korichneva I, Dhennin-Duthille I, et al. Recent Advances in Oncogenic Roles of the TRPM7 Chanzyme. Curr Med Chem. 2016;23:4092-107.

47. Dhennin-Duthille I, Gautier M, Korichneva I, Ouadid-Ahidouch H. TRPM7 involvement in cancer: a potential prognostic factor. Magnes Res. 2014;27:103-12.

48. Yin YC, Lin CC, Chen TT, Chen JY, Tsai HJ, Wang CY, et al. Clozapine induces autophagic cell death in non-small cell lung cancer cells. Cell Physiol Biochem. 2015;35:945-56.

49. Barak Y, Achiron A, Mandel M, Mirecki I, Aizenberg D. Reduced cancer incidence among patients with schizophrenia. Cancer. 2005;104:2817-21.

50. Catts VS, Catts SV, O'Toole BI, Frost AD. Cancer incidence in patients with schizophrenia and their first-degree relatives - a meta-analysis. Acta Psychiatr Scand. 2008;117:323-36.

51. Chou FH, Tsai KY, Su CY, Lee CC. The incidence and relative risk factors for developing cancer among patients with schizophrenia: a nine-year follow-up study. Schizophr Res. 2011;129:97-103.

\section{Figures}


A

B
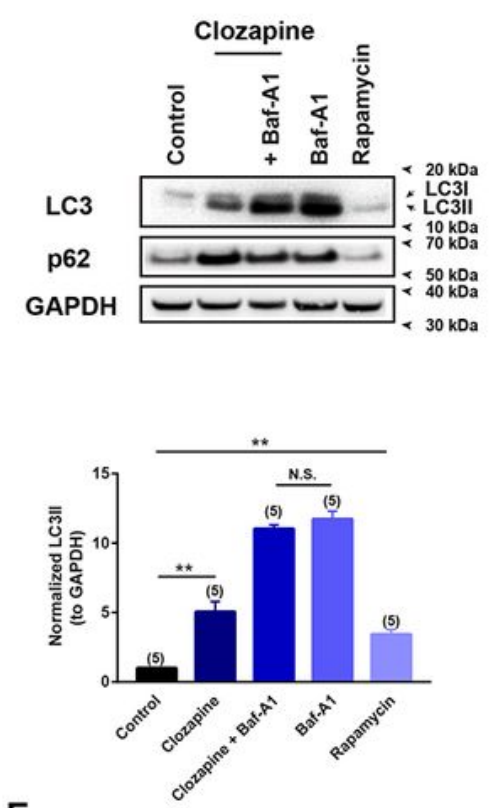

E
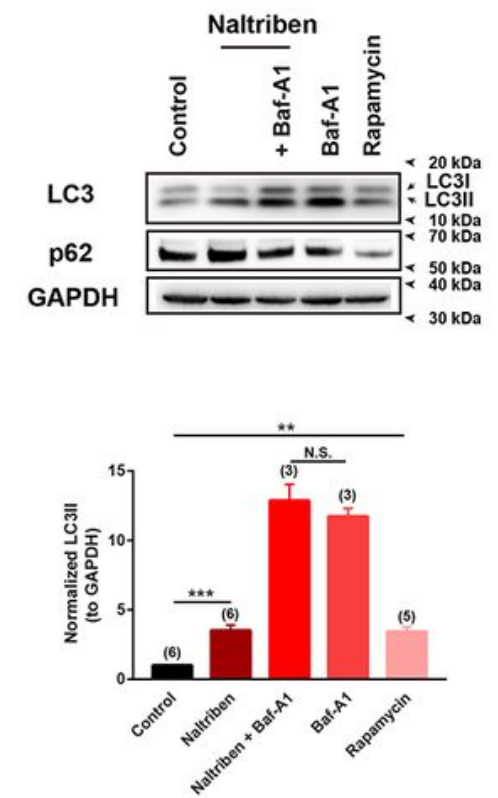

C
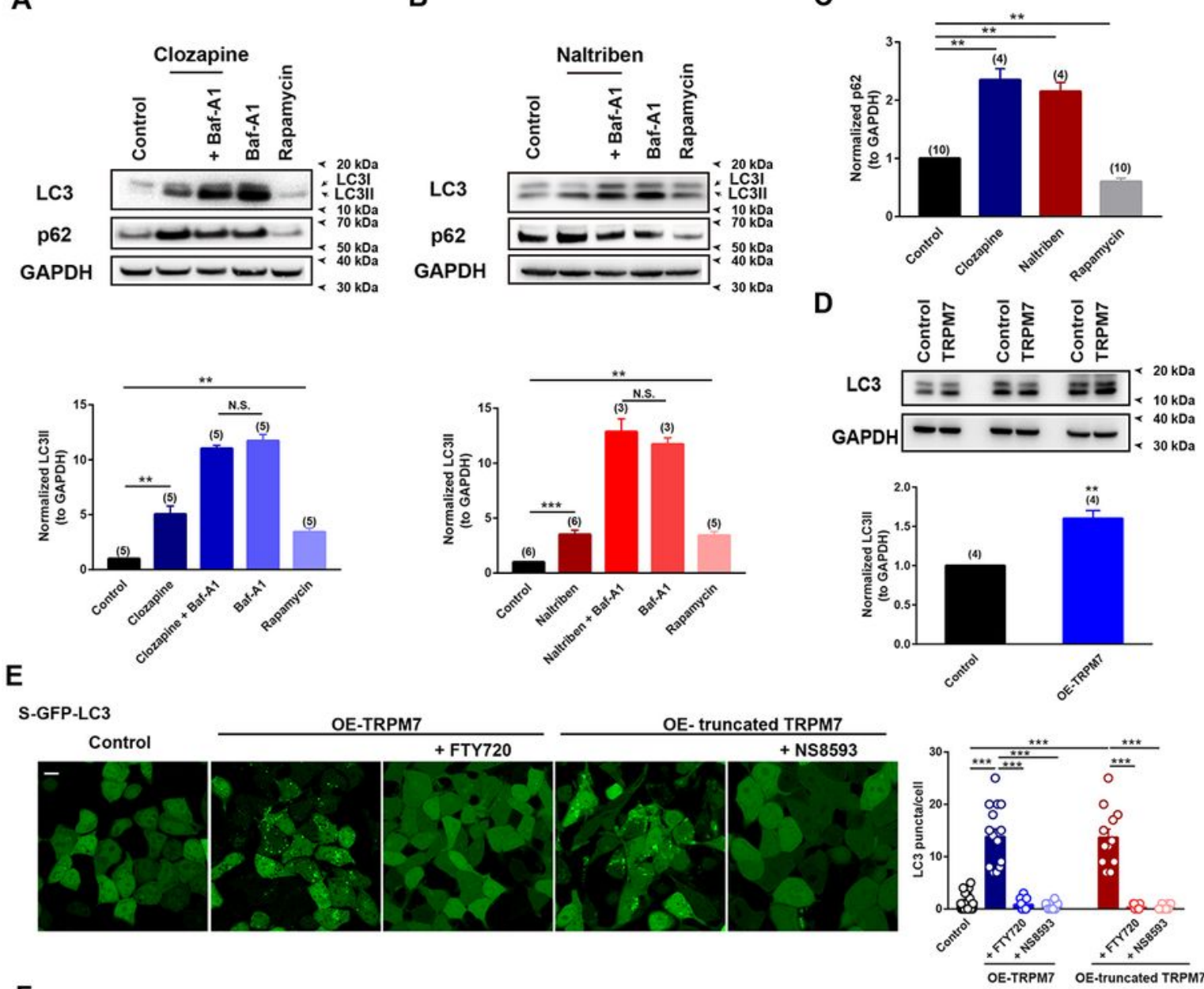

F

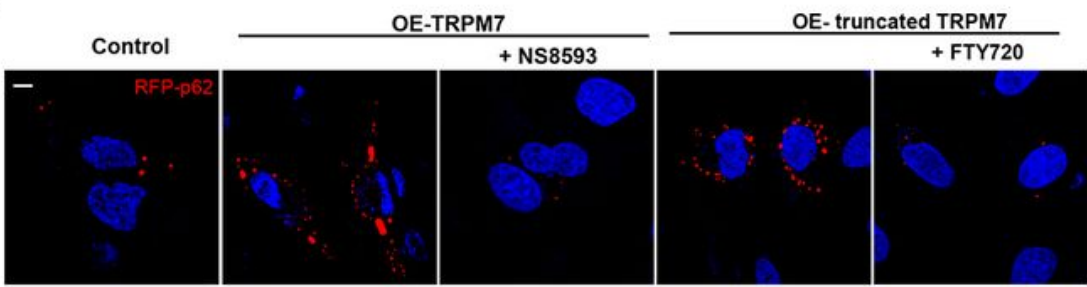

G
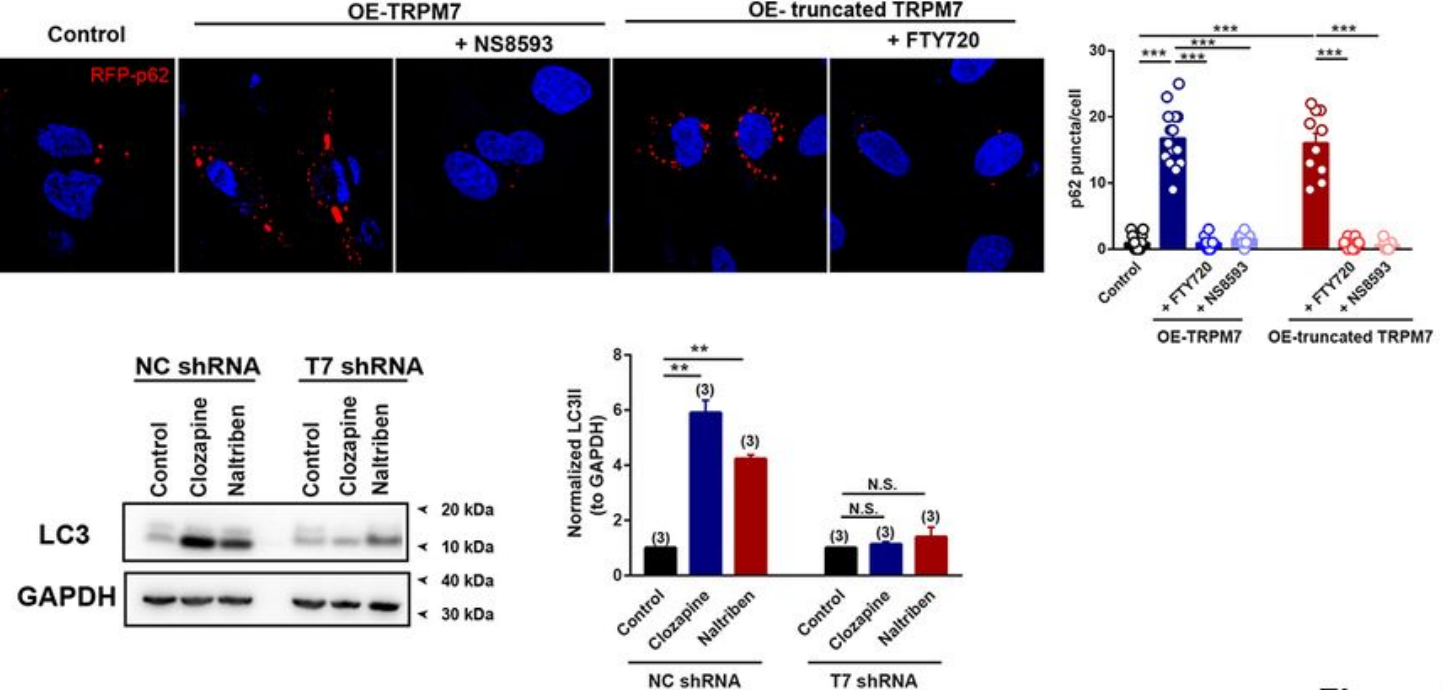

Figure 1

\section{Figure 1}

Stimulating TRPM7 channels specifically regulates autophagy. (A-C) LC3II and p62 protein levels in Hela cells were determined by western blot experiments. Clozapine (20 $\mu \mathrm{M})(\mathrm{A})$, Naltriben (5 $\mu \mathrm{M})(\mathrm{B}), \mathrm{Baf}-\mathrm{A} 1$ (1 $\mu \mathrm{M})$, and Rapamycin (50 $\mu \mathrm{M})$ were applied for $4 \mathrm{~h}$. Rapamycin was used as a positive control for measuring LC3II and p62. GAPDH was a loading control. Statistical analysis of LC3II levels (A and B) and p62 levels (C) under the treatments indicated in A and B were shown. $n=3-6$. (D) Compared with the non- 
transduced Hela cells, the basal LC3II levels were significantly increased in cells transduced with lentiTRPM7 (OE-TRPM7), as assessed by western blot experiments. Statistical analysis of LC3Il levels under control and OE-TRPM7 were followed. $n=4$. (E) Overexpressing with TRPM7 (OE-TRPM7) or truncated TRPM7 (OE-truncated TRPM7) significantly increased LC3 puncta in GFP-LC3 stably expressed HEK 293T cells (S-GFP-LC3). Increased LC3 puncta structures by OE-TRPM7 or OE-truncated TRPM7 were completely abolished by the application of FTY720 $(1 \mu \mathrm{M})$ or NS8593 $(20 \mu \mathrm{M})$. Statistical analysis was quantified from cells under different treatments from at least three independent experiment sets. Scale bar $=10 \mu \mathrm{m}$. (F) Confocal images and statistical analysis indicating that OE-TRPM7 or OE-truncated TRPM7 strongly increased puncta structures of p62 in RFP-p62-overexpressing Hela cells. Statistical analysis was quantified from cells under different treatments from at least three independent experiment sets. Scale bar $=10 \mu \mathrm{m}$. (G) Compared with Hela cells transduced with NC (negative control) shRNA, the basal LC3II levels in Hela cells transfected with T7 shRNA were not significantly altered. The effects of Clozapine $(20 \mu \mathrm{M})$ or Naltriben $(5 \mu \mathrm{M})$ on increasing LC3II levels were significantly compromised in cells transfected with T7 shRNA compared to the effects in cells transfected with NC shRNA. Treatments were for $12 \mathrm{~h}$. $\mathrm{n}=3$. Means \pm SEMs were shown in panels A-G. Significance of differences was evaluated using one-way ANOVA followed by Tukey's test. $* \mathrm{P}<0.05$; $* * \mathrm{P}<0.01$; ***P $<0.001$.

A

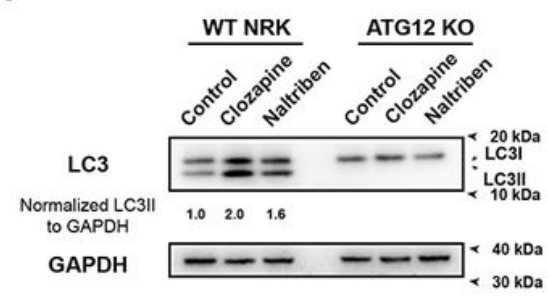

C
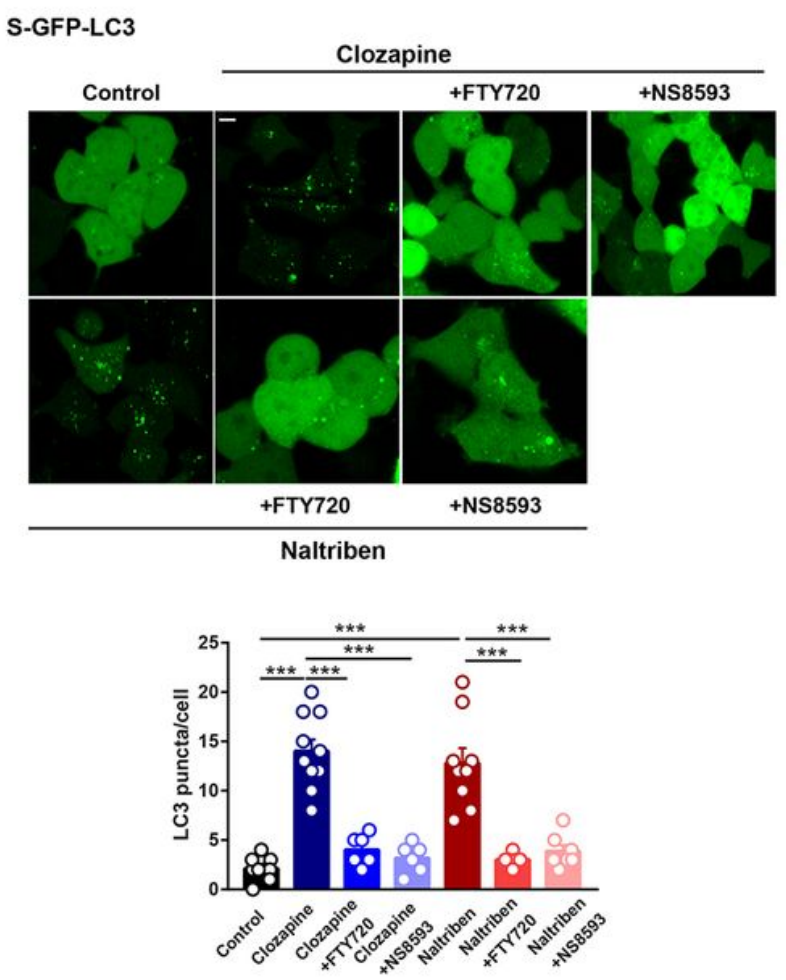

B

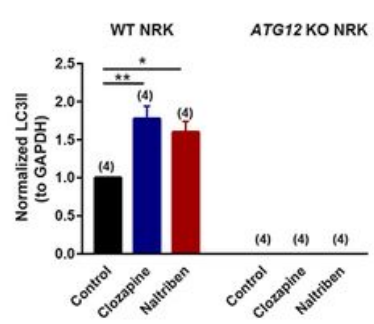

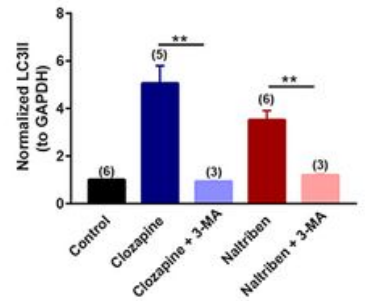

D

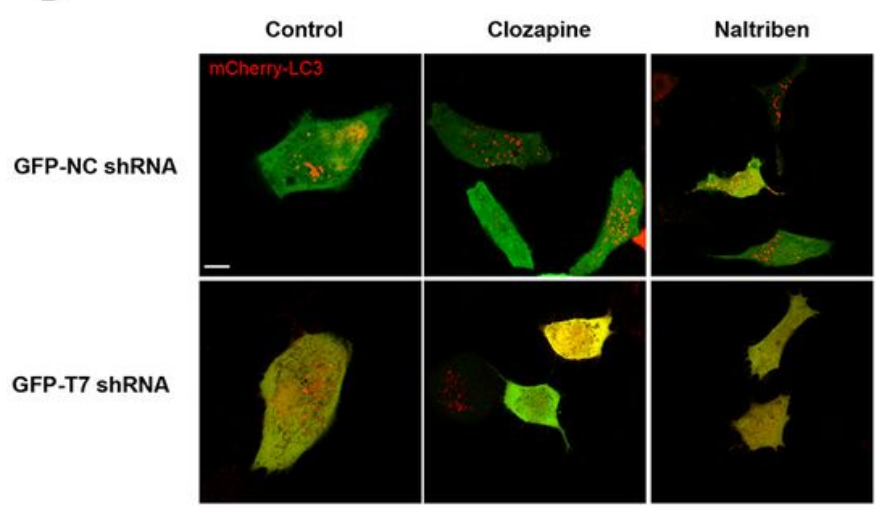

Figure 2 


\section{Figure 2}

Treatment with Clozapine or Naltriben specifically regulates autophagy by modulating TRPM7 channel activity. (A) Application of Clozapine $(20 \mu \mathrm{M})$ or Naltriben $(5 \mu \mathrm{M})$ led to significant LC3II increases in WT NRK cells, whereas neither drug induced LC3II conversion in ATG12 KO NRK cells. LC3II levels normalized to GAPDH were quantified from 4 independent experiments. (B) Pretreatment with 3-MA (10 mM) for $2 \mathrm{~h}$ strongly prevented LC3II increases by Clozapine $(20 \mu \mathrm{M}$ for $12 \mathrm{~h})$ or Naltriben $(5 \mu \mathrm{M}$ for $12 \mathrm{~h})$ treatment in Hela cells. Statistical analysis of LC3II levels under the treatments indicated were displayed. n=3-6. (C) Representative images and statistical analysis showing that the treatment of Clozapine $(20 \mu \mathrm{M})$ or Naltriben $(5 \mu \mathrm{M})$ induced overt GFP-LC3 puncta structures in GFP-LC3 stably expressed HEK 293T cells (S-GFP-LC3). Co-application of FTY720 $(1 \mu \mathrm{M})$ or NS8593 $(20 \mu \mathrm{M})$ profoundly reduced puncta structures induced by Clozapine or Naltriben. All treatments were for $4 \mathrm{~h}$. Statistical analysis was quantified from cells under different treatments from at least three independent experiment sets. Scale bar $=10 \mu \mathrm{m}$. (D) Representative images and statistical analysis displaying that the treatment of Clozapine (20 $\mu \mathrm{M}$ for $4 \mathrm{~h})$ or Naltriben $(5 \mu \mathrm{M}$ for $4 \mathrm{~h})$ triggered mCherry-LC3 puncta structures in mCherry-LC3 and GFP-NC shRNA transiently co-expressed Hela cells. By contrast, LC3 puncta structures were barely observed under the treatment of Clozapine $(20 \mu \mathrm{M})$ or Naltriben $(5 \mu \mathrm{M})$ in co-expressing mCherry-LC3 and GFP-T7 shRNA Hela cells. Statistical analysis was quantified from cells under different treatments from at least three independent experiment sets. Scale bar $=10 \mu \mathrm{m}$. Means \pm SEMs were shown in panels A-D. Significance of differences was evaluated using one-way ANOVA followed by Tukey's test. ${ }^{\star} \mathrm{P}<0.05$; ${ }^{\star \star} \mathrm{P}<0.01$; $* \star \star \mathrm{P}$ $<0.001$. 
A

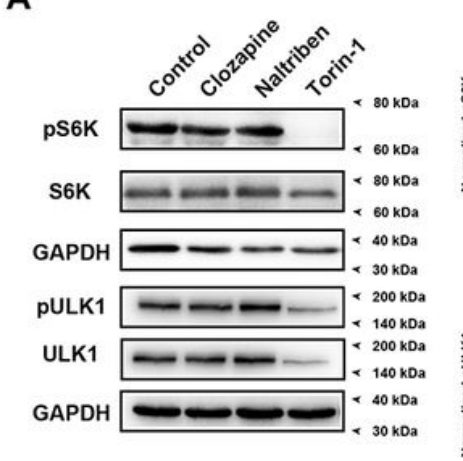

C
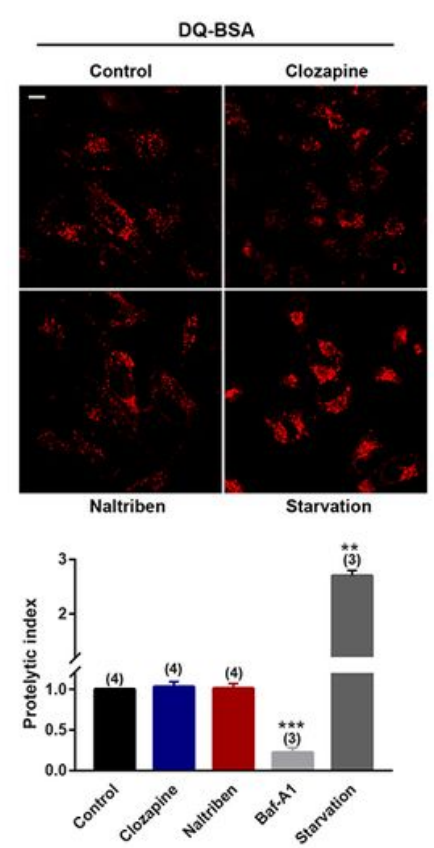

B

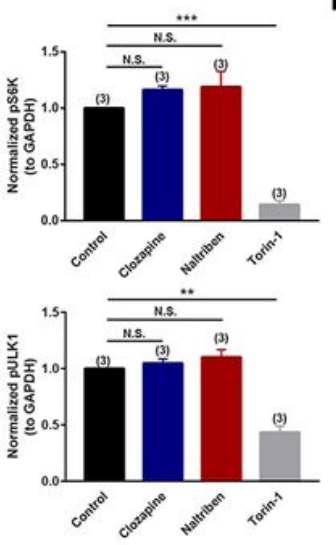

B
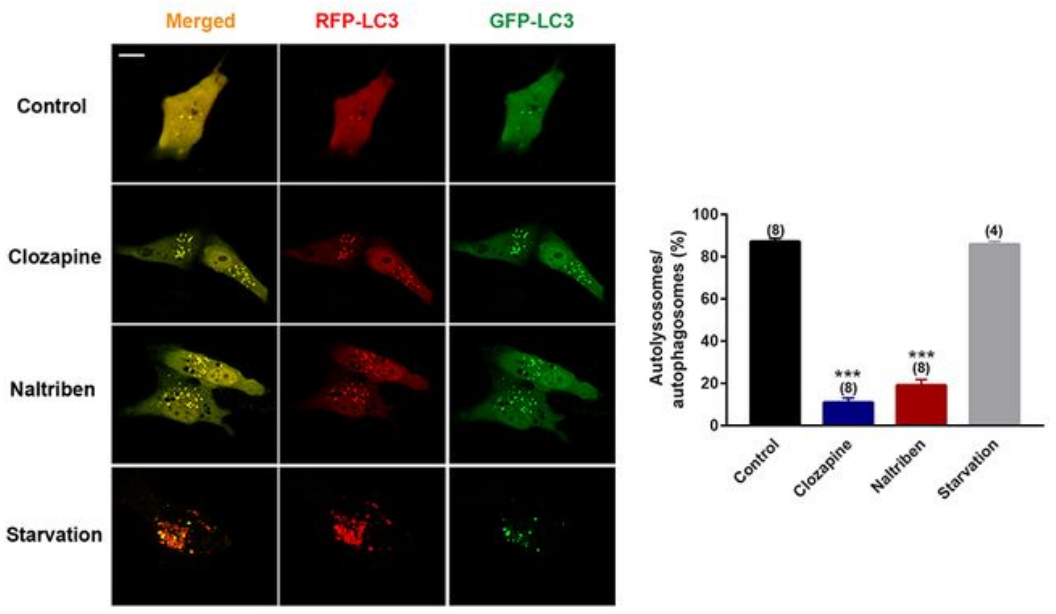

D
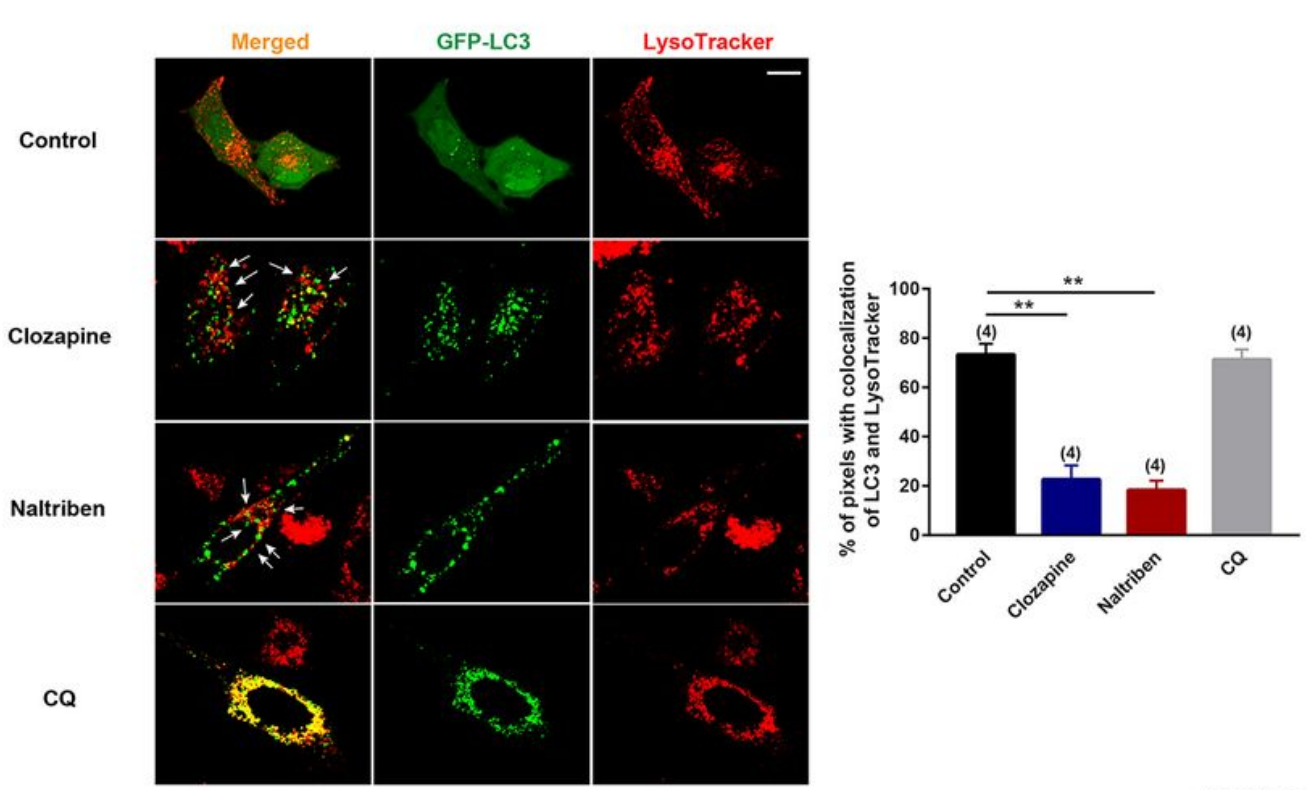

Figure 3

\section{Figure 3}

Activation of TRPM7 inhibits autophagy by disrupting fusion of autophagosomes and lysosomes. (A) S6K, Phosphorylated S6K (pS6K at T389), ULK1, phosphorylated ULK1 (pULK1 at S757) levels in Hela cells under the control, Clozapine $(20 \mu \mathrm{M})$, Naltriben $(5 \mu \mathrm{M})$ and Torin-1 $(1 \mu \mathrm{M})$ were determined using western blot experiments. Torin- 1 was used as a positive control to measure pS6K and pULK1. S6K and ULK1 levels were measured and served as loading controls along with GAPDH. All treatments were for 12 h. $n=3$. (B) Treatment with Clozapine $(20 \mu \mathrm{M})$ or Naltriben $(5 \mu \mathrm{M})$ led to equal numbers of green puncta and red puncta, while starvation (AA + FBS free; bottom panels) induced much greater numbers of red puncta over green puncta in Hela cells transiently expressing tandem GFP-RFP-LC3. Scale bar $=10 \mu \mathrm{m}$. All treatments were for $4 \mathrm{~h}$. Ratio quantification of GFP puncta to RFP puncta under the indicated conditions outlined. $n=4-8$. (C) Representative images of DQ-red-BSA in Hela cells under conditions of control, starvation (AA + FBS free), Clozapine $(20 \mu \mathrm{M})$, Naltriben $(5 \mu \mathrm{M})$, and Baf-A1 (1 $\mu \mathrm{M})$, respectively. All treatments were for $4 \mathrm{~h}$. Scale bar $=10 \mu \mathrm{m}$. Proteolytical index of DQ-BSA assay were normalized under conditions indicated. Typically 25- 80 cells were counted for quantification from 3-4 independent 
experiments. (D) Representative confocal images and statistical analysis of co-localization between autophagosomes (green puncta) and lysosomes (red puncta) under control, Clozapine (20 $\mu \mathrm{M})$, Naltriben $(5 \mu \mathrm{M})$, and CQ $(10 \mu \mathrm{M})$ treatment in GFP-LC3 transiently expressed and LysoTracker stained Hela cells. The arrows indicate that autophagosomes (GFP-LC3 puncta) were adjacent to, but not fused to, lysosomes stained by LysoTracker. Typically 6-20 cells were counted for quantification from at least three independent experiments. All treatments were for $4 \mathrm{~h}$. Scale bar $=10 \mu \mathrm{m}$. Means \pm SEMs were shown in panels A-D. Significance of differences was evaluated using one-way ANOVA followed by Tukey's test. $* * \mathrm{P}<0.01 ; * * * \mathrm{P}<0.001$.

A

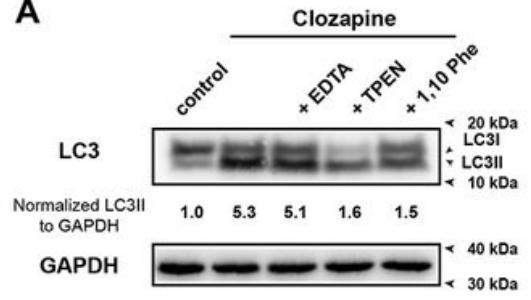

D
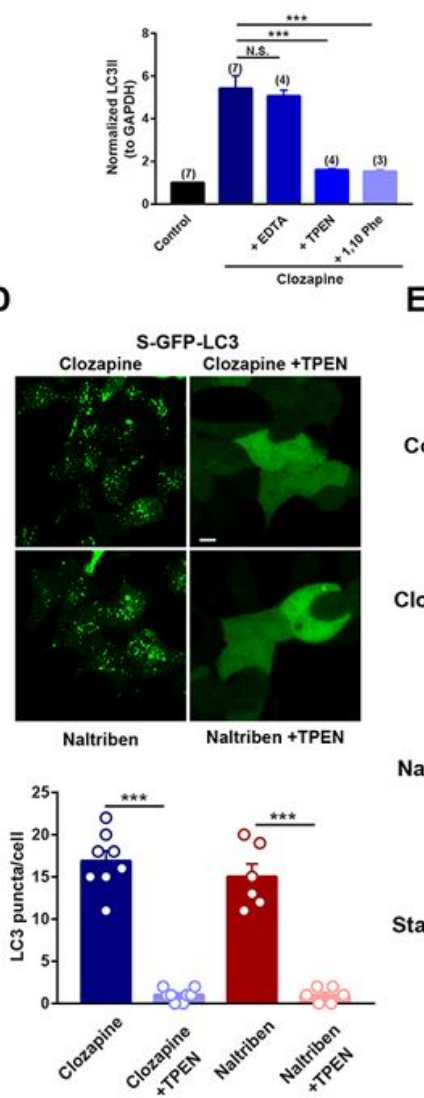

\section{E}

B
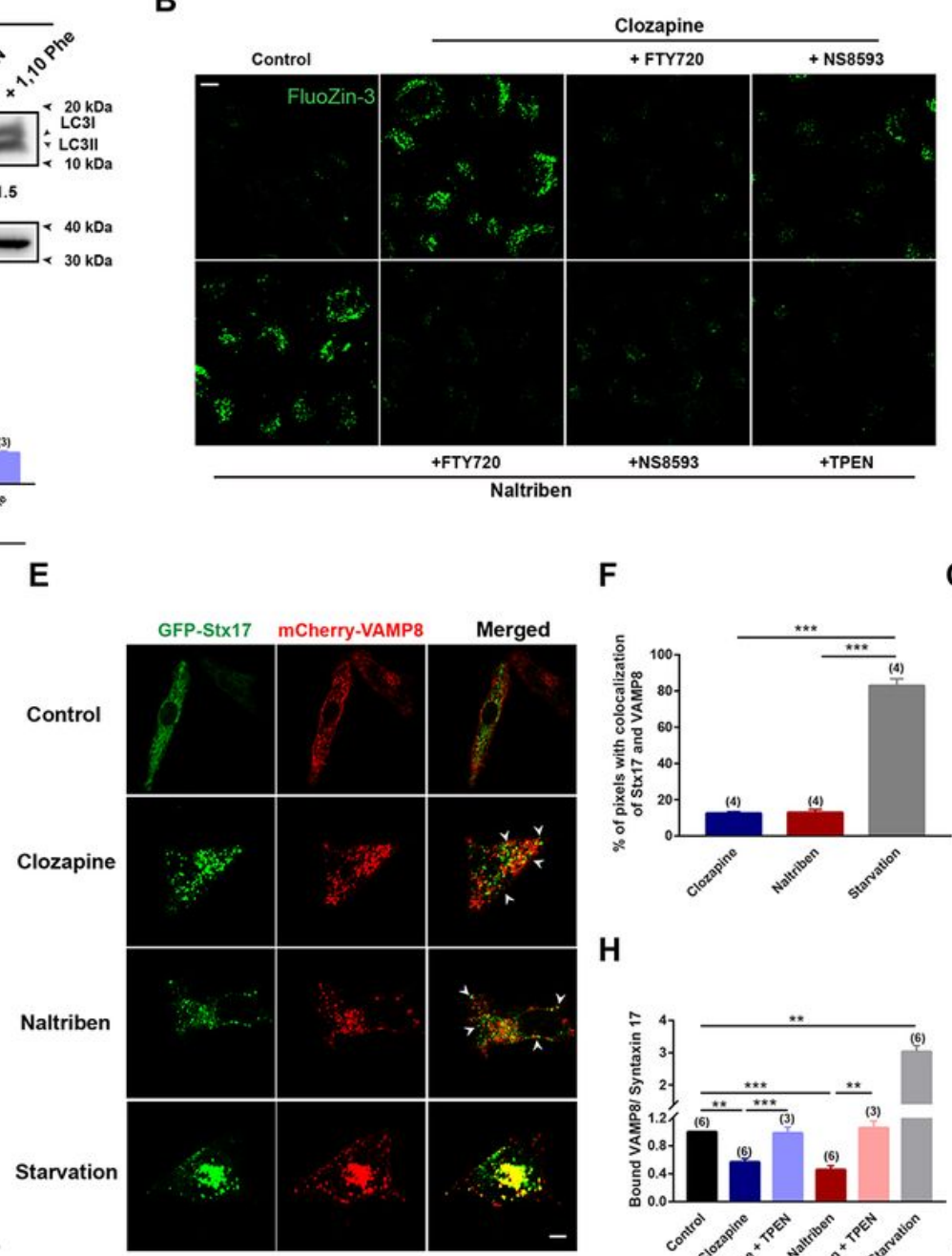

F

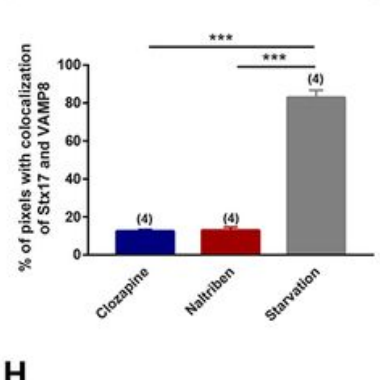

H

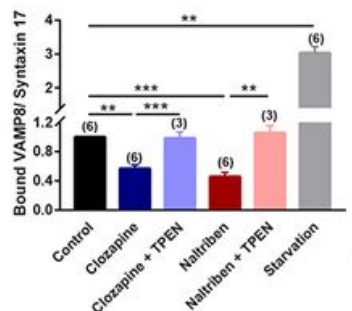

C

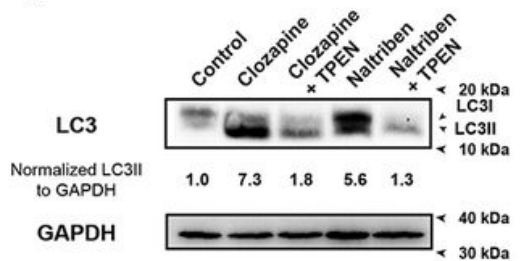

G
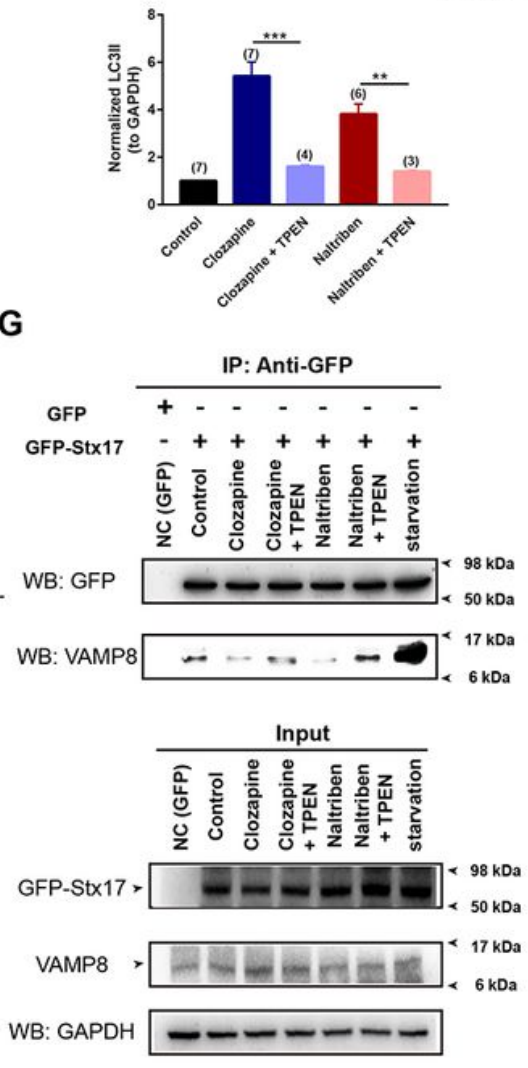

Figure 4

Figure 4

Activation of TRPM7 mediates zinc influx to the cytosol, which in turn eliminates the interaction between Stx17 in the autophagosome and VAMP8 in the lysosome. (A) LC3II levels in Hela cells under control, Clozapine $(20 \mu \mathrm{M})$, Clozapine $(20 \mu \mathrm{M})+$ EDTA $(5 \mathrm{mM})$, Clozapine + TPEN $(10 \mu \mathrm{M})$ and Clozapine $+1,10$ Phe $(500 \mu \mathrm{M})$ treatment were determined with western blot experiments. All treatments were for $4 \mathrm{~h}$. Normalized LC3II levels (to GAPDH) under the treatments indicated were quantified from 3-7 independent experiments. (B) Cytosolic zinc concentrations under the control, Clozapine (20 $\mu \mathrm{M})$, Clozapine + FTY720 
$(1 \mu \mathrm{M})$, Clozapine + NS8593 $(20 \mu \mathrm{M})$, Naltriben $(5 \mu \mathrm{M})$, Naltriben + FTY720 $(1 \mu \mathrm{M})$, Naltriben + NS8593 $(20 \mu \mathrm{M})$ and Naltriben + TPEN $(10 \mu \mathrm{M})$, were evaluated by zinc staining with a FluoZin-3 probe. All treatments were for $4 \mathrm{~h}$. Scale bar $=10 \mu \mathrm{m}$. (C) Application of TPEN $(10 \mu \mathrm{M})$ completely abrogated the increase in LC3II levels in Hela cells resulting from Clozapine $(20 \mu \mathrm{M})$ or Naltriben $(5 \mu \mathrm{M})$. All treatments were for $12 \mathrm{~h}$. Statistical analysis or LC3II levels under the treatments indicated were quantified from 3-7 independent experiments. (D) GFP-LC3 Puncta structures triggered by the treatment of Clozapine $(20 \mu \mathrm{M})$ or Naltriben $(5 \mu \mathrm{M})$ were markedly reduced following application of TPEN $(10 \mu \mathrm{M})$ in GFP-LC3 stably expressed HEK 293T cells (S-GFP-LC3). All treatments were for $4 \mathrm{~h}$. Statistical analysis was quantified from cells under different treatments from at least three independent experiment sets. Scale bar $=10 \mu \mathrm{m}$. $(E-F)$ Representative confocal images (E) and statistical analysis (F) (typically $n=4-8$ cells from at least three independent experiments) displaying co-localization of GFP-Stx17 and mCherry-VAMP8 in GFPStx17 and mCherry-VAMP transiently expressed Hela cells under control, Clozapine ( $20 \mu \mathrm{M})$, Naltriben $(5$ $\mu \mathrm{M})$ and starvation (AA free + FBS free) conditions. All treatments were for $4 \mathrm{~h}$. Scale bar $=10 \mu \mathrm{m}$. (G-H) Co-IP assays revealing that the interaction between Stx17 and VAMP8 in Hela cells was significantly reduced by the treatment of Clozapine $(20 \mu \mathrm{M})$ or Naltriben $(10 \mu \mathrm{M})$ compared to control, whereas starvation (AA and FBS free) increased the interaction. Application of TPEN (10 $\mu \mathrm{M})$ significantly rescued the eliminated interaction between Stx17 and VAMP8 by Clozapine or Naltriben. All treatments were for $12 \mathrm{~h}$. GFP-Stx17 was immunoprecipitated by GFP antibody and endogenous VAMP8 were co-IPed with Stx17. VAMP8 was then normalized to total GFP-Stx17. $n=3-6$. Means \pm SEM was shown in panels A, C, D, $\mathrm{F}$ and $\mathrm{H}$. Significance of differences was evaluated using one-way ANOVA followed by Tukey's test. **P $<0.01$; $* \star * P<0.001$. 
A

- Control $20 \mu \mathrm{M}$ Clozapine

- $10 \mu \mathrm{M}$ Clozapine $40 \mu \mathrm{M}$ Clozapine

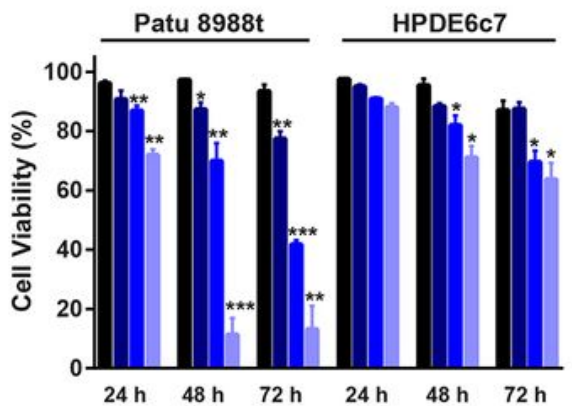

C

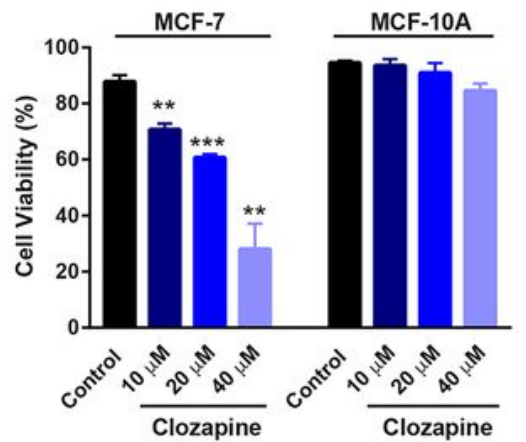

E

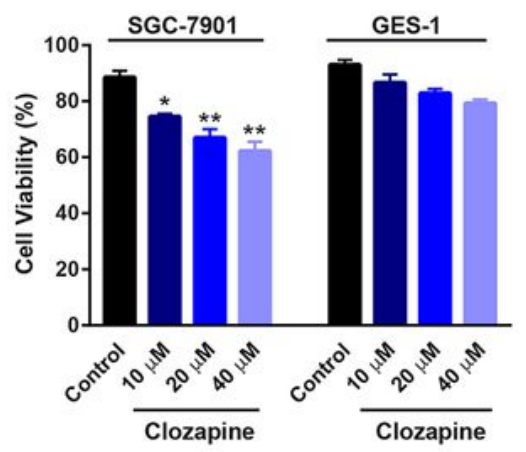

G

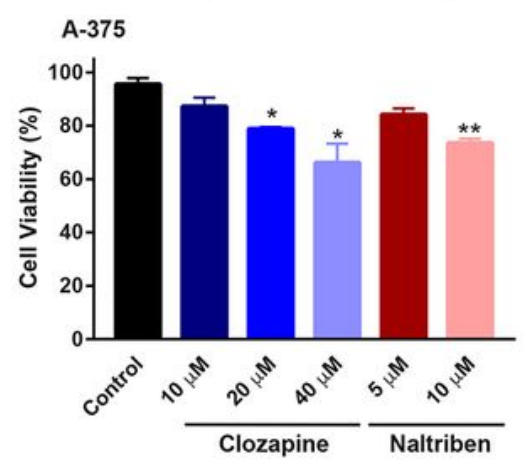

B

Control $\square$ ㅆM Naltriben $\square 10 \mu \mathrm{M}$ Naltriben

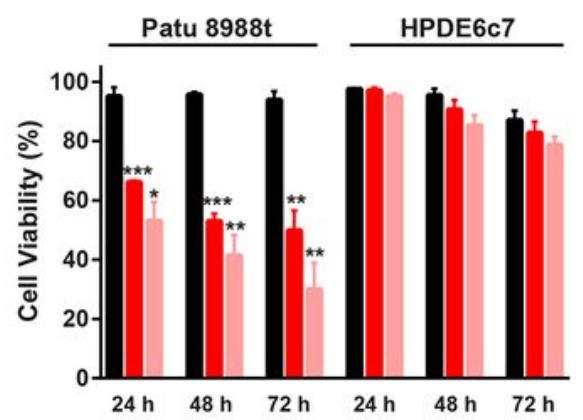

D

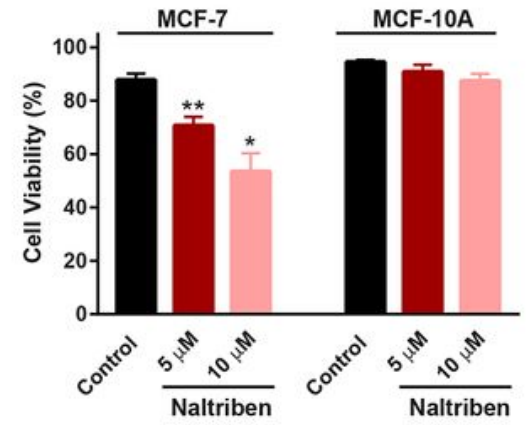

F

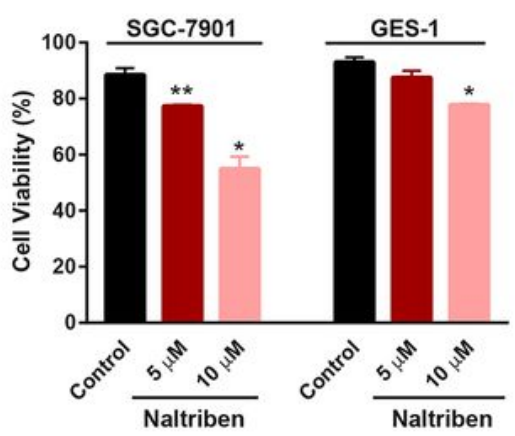

H

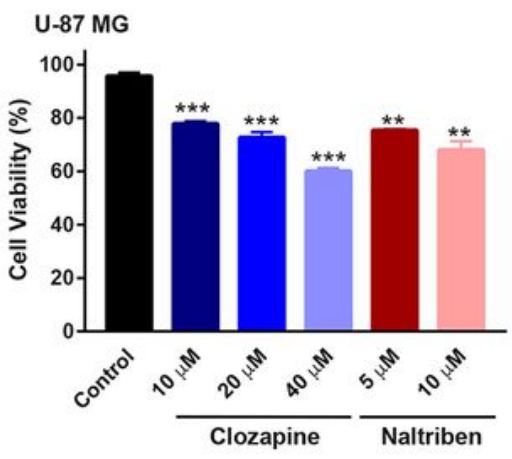

Figure 5

\section{Figure 5}

Stimulating TRPM7 triggers cell death in a variety of cancer cells with limited effects on the corresponding normal cells. (A-H) Cell viability of different cells including Patu 8988t and HPDE6c7 cells (A-B), MCF-7 and MCF-10A cells (C-D), SGC-7901 and GES-1 cells (E-F), A-375 cells (G), and U-87 MG cells $(\mathrm{H})$, were measured by Trypan Blue assay and compared between control and with treatment of Clozapine or Naltriben for the various concentrations and periods indicated. $n=3-15$. Means \pm SEMs were 
shown in all panels. Significance of differences was evaluated using one-way ANOVA followed by Tukey's test. * $\mathrm{P}<0.05 ;{ }^{* \star} \mathrm{P}<0.01 ; * \star \star * P<0.001$.

A

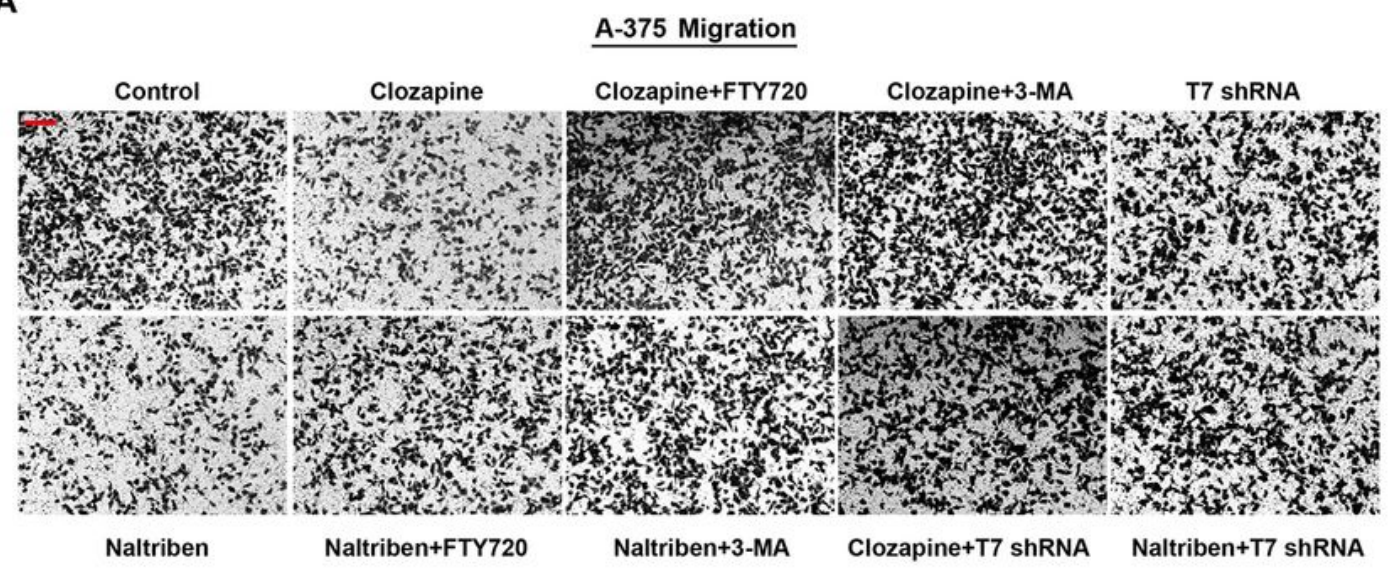

B

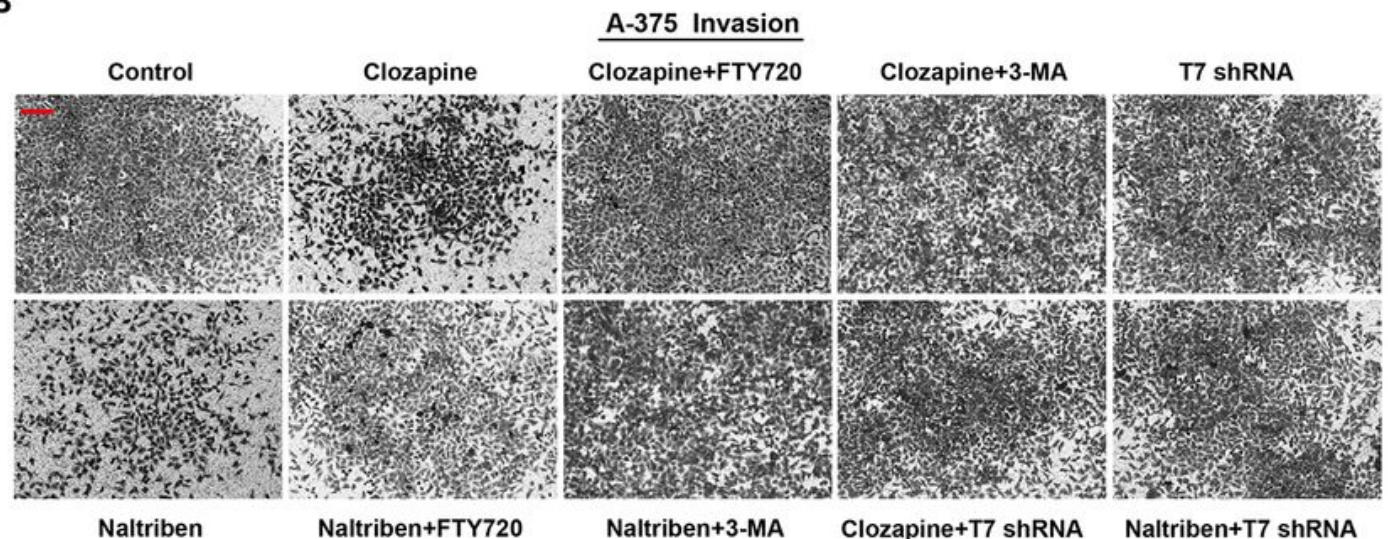

C

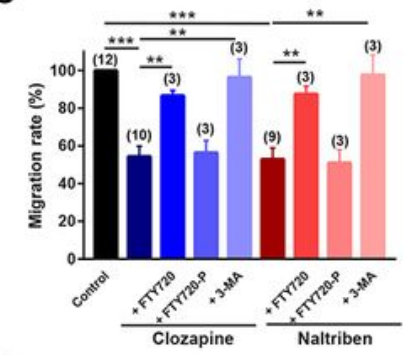

$\mathbf{F}$

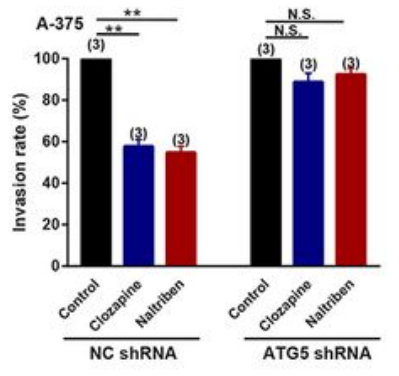

D

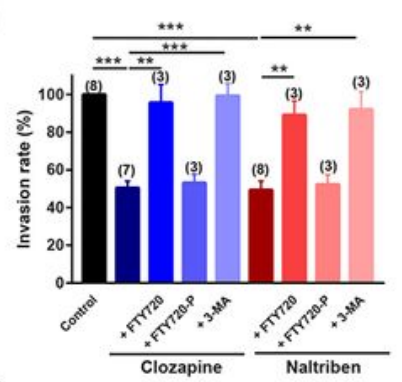

G

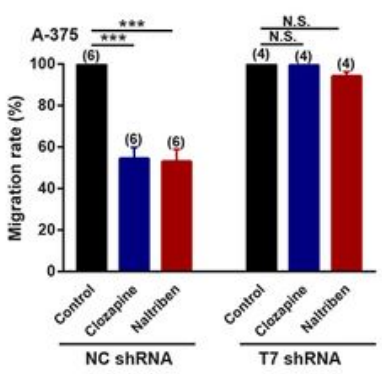

E

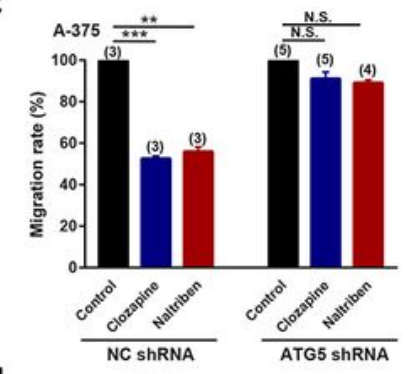

H

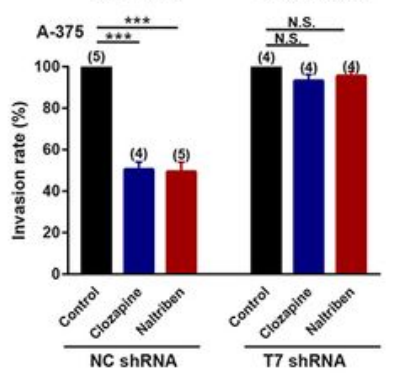

Figure 6

Figure 6

Stimulating TRPM7 suppresses cancer metastasis in a variety of cancer cells. (A-B) Representative images showing migrated (A) or invaded (B) A-375 cells stained with $0.5 \%$ crystal violet solution under conditions of control, Clozapine $(20 \mu \mathrm{M})$, Clozapine with FTY720 (1 $\mu \mathrm{M})$, Clozapine with pretreatment of 
3-MA (10 mM for $2 \mathrm{~h})$, Naltriben $(5 \mu \mathrm{M})$, Naltriben with FTY720 $(1 \mu \mathrm{M})$, and Naltriben with pretreatment of 3-MA as assessed using transwell assays. All treatments were for $16-24 \mathrm{~h}$. Scale bar $=1 \mathrm{~cm}$. (C-D) Migration rate (OD600x is normalized to OD600control) (C) and invasion rate (D) of A-375 cells were summarized under the different conditions indicated in $A$ and $B$, respectively. The results with FTY720-P were included as a negative control. Quantified results were collected from at least three independent experiments (n=3-12). (E-F) Knockdown of ATG5 significantly rescued suppressed migration $(E)$ and invasion (F) of A-375 cells regulated by the treatment of Clozapine $(20 \mu \mathrm{M})$ or Naltriben $(5 \mu \mathrm{M})$. n=3-5. (G$\mathrm{H})$ Migration rate $(\mathrm{G})$ and invasion rate $(\mathrm{H})$ under the treatment of Clozapine $(20 \mu \mathrm{M})$ or Naltriben $(5 \mu \mathrm{M})$ were compared between A-375 cells transduced with the NC shRNA and A-375 cells transduced with the T7 shRNA. $n=4-6$. Means \pm SEMs were shown in panels $C-H$. Significance of differences was evaluated using one-way ANOVA followed by Tukey's test. ** $\mathrm{P}<0.01$; $* \star \star \mathrm{P}<0.001$.

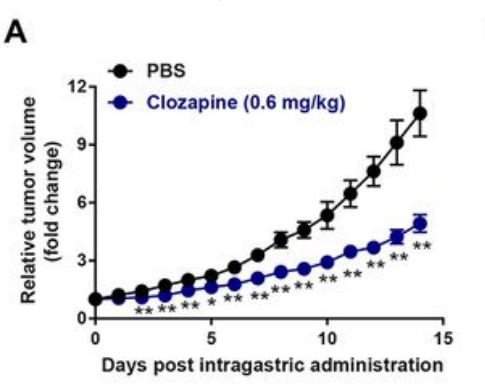

E

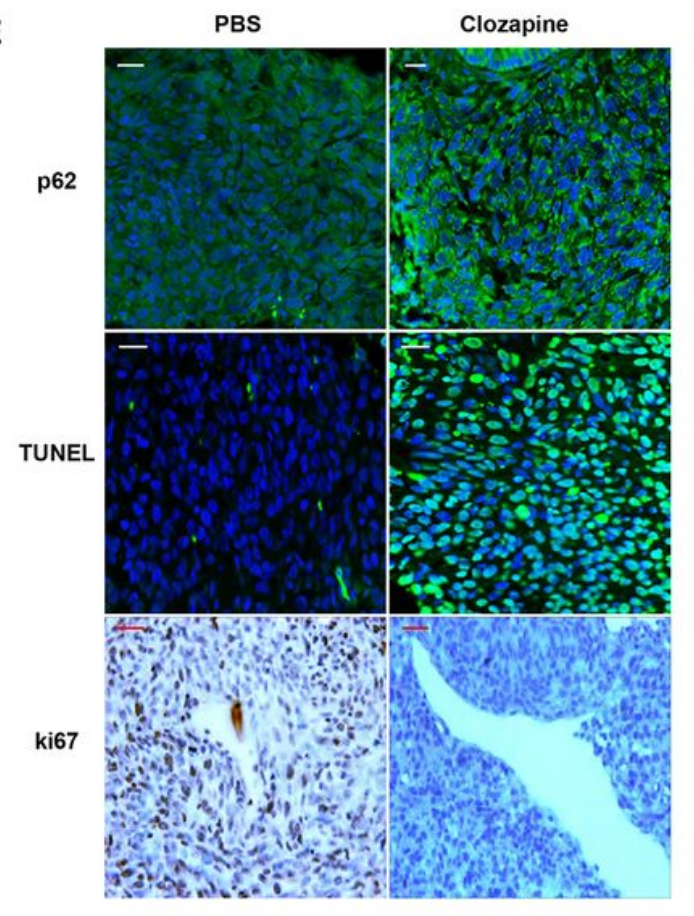

B

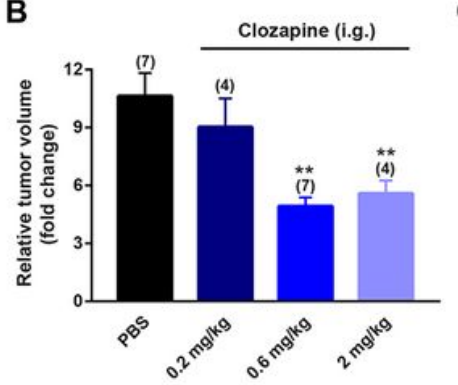

$\mathrm{F}$

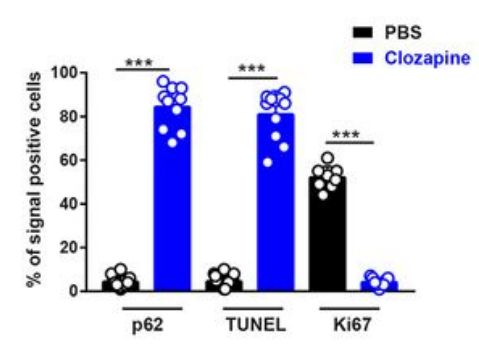

H

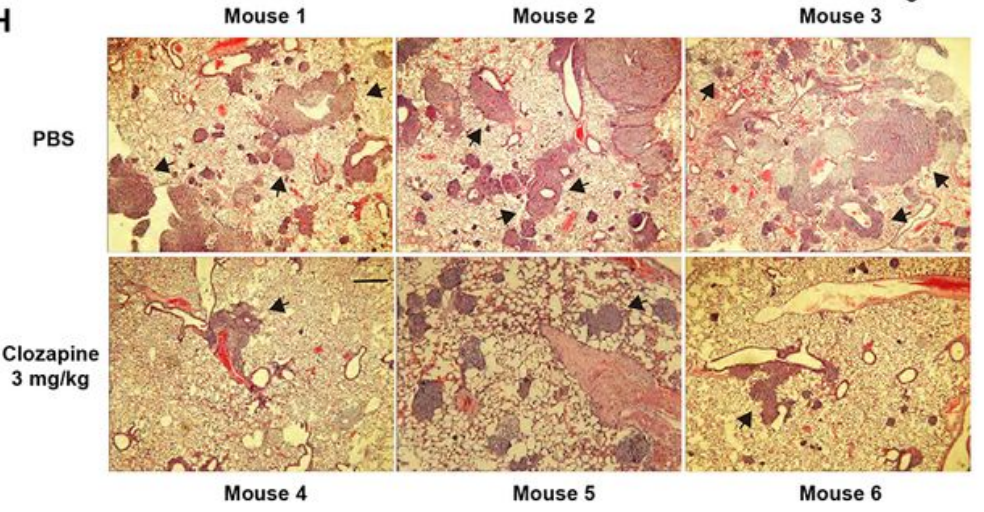

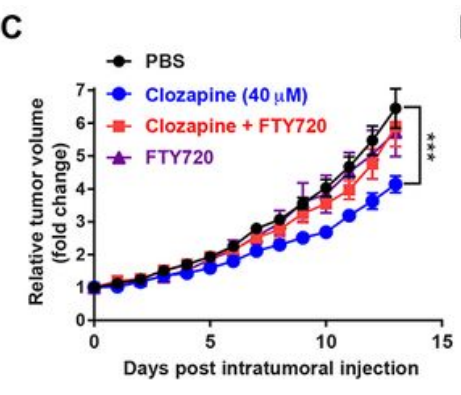

G
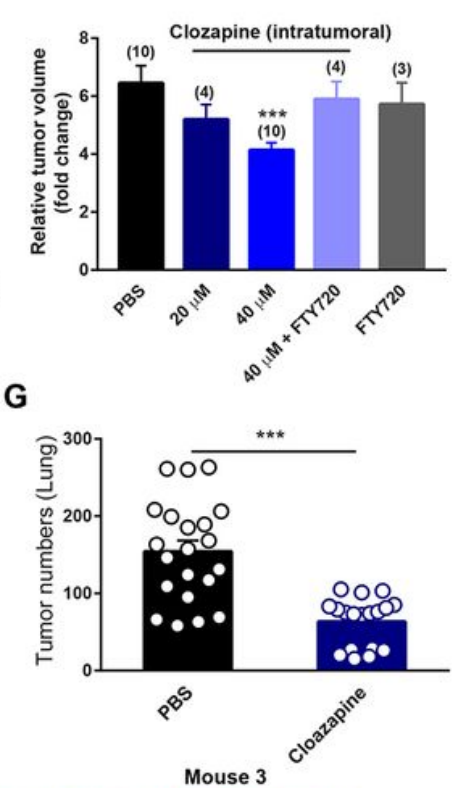

Mouse 6

Figure 7

\section{Figure 7}

Stimulating TRPM7 with administration of Clozapine suppresses tumor growth and metastasis in vivo. (A) Patu 8988t xenograft growth of control group and Clozapine group by daily intragastric administration (i.g.) of PBS or Clozapine $(0.6 \mathrm{mg} / \mathrm{kg}$ ) was monitored over the course of 14 days. $\mathrm{n}=7$. (B) Final relative tumor volumes of control group and Clozapine groups with different doses administration 
(i.g.) of Clozapine indicated were compared. (C) Patu 8988t xenograft growth of different groups was monitored over the course of 14 days, under daily intratumoral injection of PBS, Clozapine $(40 \mu \mathrm{M})$, Clozapine $(40 \mu \mathrm{M})+$ FTY720 $(10 \mu \mathrm{M})$, and FTY720 $(10 \mu \mathrm{M})$, respectively. $\mathrm{n}=3-10$. (D) Final relative tumor volumes of different groups under conditions (intratumarol injection) indicated in c were summarized. $\mathrm{n}=3-10$. (E) Representative immunofluorescence and immunohistology staining confirming that administration of Clozapine (i.g. $0.6 \mathrm{mg} / \mathrm{kg}$ ) induced significant autophagy inhibition, cell death, and delayed proliferation in tumors, supported by increased levels of p62 and Tunel and significantly decreased signals of ki67, respectively. Scale bar $=20 \mu \mathrm{m}$. (F) Quantification of percentage of cells positive for p62, Tunel, and ki67 per field of tissue sections from tumors of PBS and Clozapine groups, respectively. $n=8-20$ sections from at least 6 animals from each group. (G) Mean numbers of lung metastases at 3 weeks in mice with parental tumors (A-375) that daily received PBS or Clozapine (3 $\mathrm{mg} / \mathrm{kg}$ ) i.p. following tumor implantation $(p=0.0000449) . n=18-21$ sections from 6 animals from each group. $(H)$ Representative H\&E-stained sections of lungs of mice injected with A-375 cells from PBS and Clozapine groups, respectively. PBS or Clozapine $(3 \mathrm{mg} / \mathrm{kg})$ was daily i.p. administrated for 3 weeks. Arrows denote lung metastases. Scale bar $=1 \mathrm{~mm}$. Error bars indicate Mean \pm SEMs in panels A, B, C, D, F and $\mathrm{G}$. Statistical comparisons were made using one-way ANOVA followed by Tukey's test or student $\mathrm{t}$ test. ${ }^{*} \mathrm{P}<0.05 ;{ }^{* * \mathrm{P}}<0.01 ; * \star * \mathrm{P}<0.001$.

A

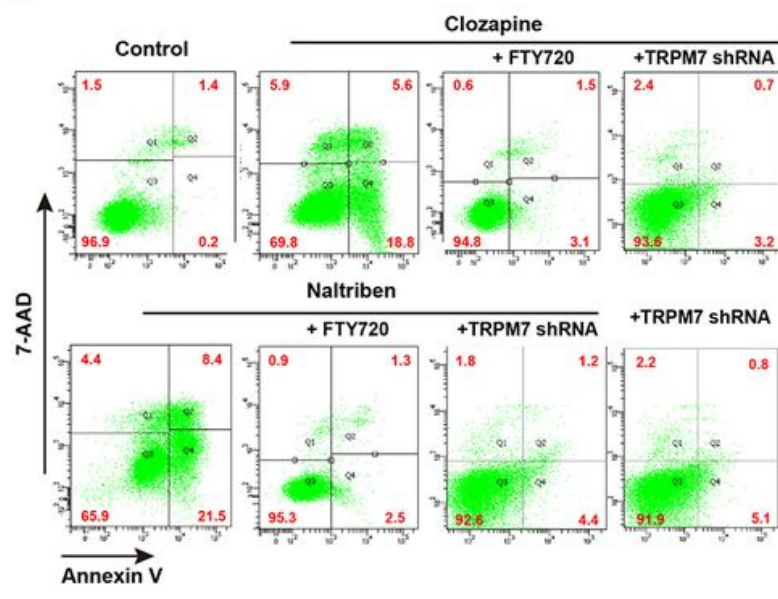

C

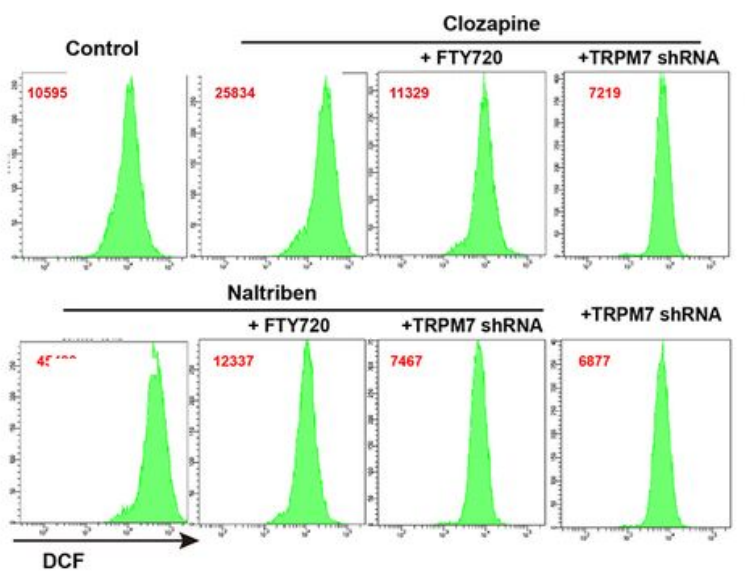

B

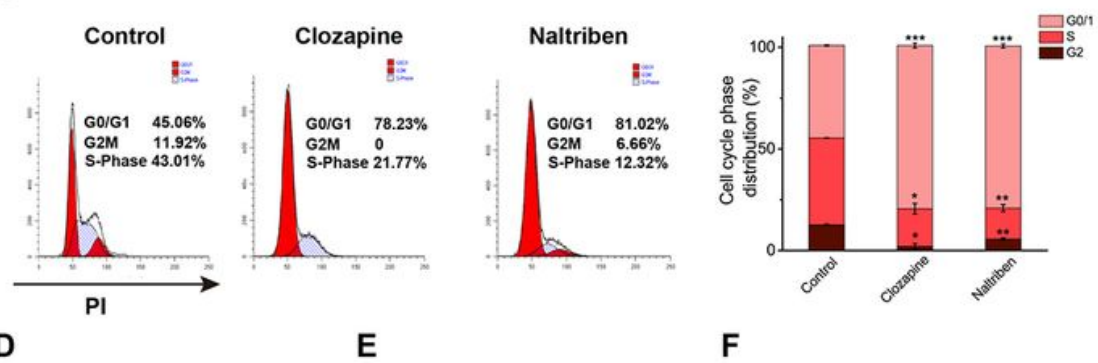

E
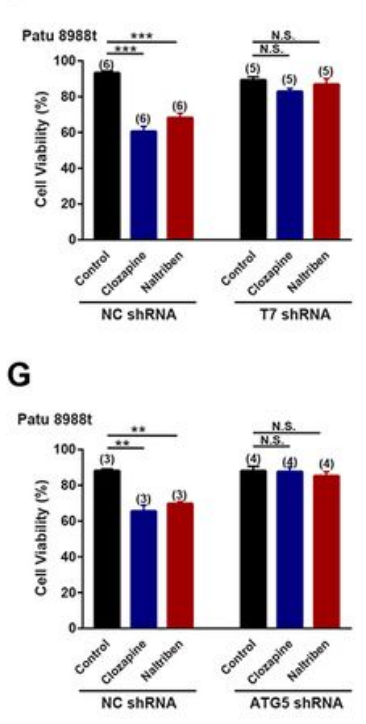
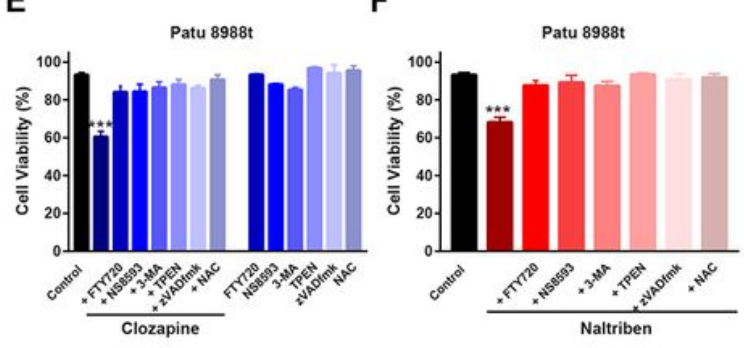

H

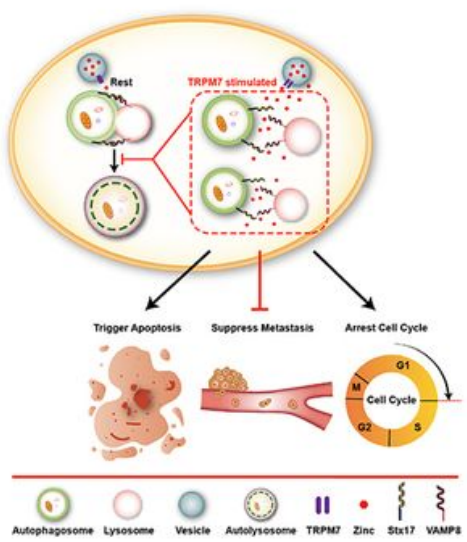

Figure 8 


\section{Figure 8}

Autophagy inhibition by stimulating TRPM7 triggers cancer cells death by evoking apoptosis, cell cycle arrest, and ROS elevation. (A-C) Flow cytometry results displaying changes in apoptosis (A), cell cycles (B), and ROS levels (C) following different treatments indicated in Patu 8988t cells, stained by Annexin V/7-AAD, PI (propidium iodide), and H2DCFDA. Clozapine $(20 \mu \mathrm{M})$, Naltriben $(5 \mu \mathrm{M})$ and FTY720 $(1 \mu \mathrm{M})$ were used in flow cytometry analysis. All treatments were for $48 \mathrm{~h}$. (D) The treatments of Clozapine (20 $\mu \mathrm{M})$ or Naltriben $(5 \mu \mathrm{M})$ triggered overt cell death in Patu $8988 \mathrm{t}$ cells transduced with the NC shRNA, whereas neither of them led to significant cell death in cells transduced with the T7 shRNA. Cell viability was measured using a Trypan Blue assay. All treatments were for 48 h. $n=5-8$. (E) Cell viability of Patu $8988 \mathrm{t}$ cells shown under the conditions of control, Clozapine $(20 \mu \mathrm{M})$, Clozapine + FTY720 $(1 \mu \mathrm{M})$, Clozapine + NS8593 $(20 \mu \mathrm{M})$, Clozapine + 3-MA (10 mM pretreated for $2 \mathrm{~h})$, Clozapine + TPEN $(1 \mu \mathrm{M})$, Clozapine + zVADfmk $(20 \mu \mathrm{M})$, Clozapine + NAC $(5 \mathrm{mM})$. All treatments were for $48 \mathrm{~h}$. $\mathrm{n}=3-15$. (F) Cell viability of Patu 8988t cells were shown under conditions of control, Naltriben $(5 \mu \mathrm{M})$, Naltriben + FTY720 $(1 \mu \mathrm{M})$, Naltriben + NS8593 $(20 \mu \mathrm{M})$, Naltriben + 3-MA (10 mM pretreated for $2 \mathrm{~h})$, Naltriben + TPEN (1 $\mu \mathrm{M})$, Naltriben + zVADfmk $(20 \mu \mathrm{M})$, Naltriben + NAC (5 mM). $\mathrm{n}=4-15$. (G) Knockdown of ATG5 markedly compromised the effects of Clozapine and Naltriben on triggering cell death in Patu 8988t cells. $n=3-4$. (H) A working model illustrates that stimulated TRPM7 releases intracellular zinc to the cytosol, which eliminates the interaction between Stx17 and VAMP8. As a result, the abolished interaction disrupts the fusion between autophagosomes and lysosomes and ultimately inhibits the autophagy process.

Functionally, TRPM7-mediated autophagy inhibition suppresses cancer cell growth and metastasis by evoking apoptosis and cell cycle arrest. Means \pm SEMs were shown in panels D, E, F and G. Significance of differences was evaluated using one-way ANOVA followed by Tukey's test. ${ }^{\star \star} P<0.01$; ${ }^{\star \star *} P<0.001$.

\section{Supplementary Files}

This is a list of supplementary files associated with this preprint. Click to download.

- SupplementalFigures1.pdf 